\title{
Cultural extension of Ginkgo biloba L. in Slovakia
}

\author{
Katarína Ražná*, Pavel Hrubík \\ Slovak University of Agriculture in Nitra, Faculty of Agrobiology and Food Resources, \\ Institute of Plant and Environmental Sciences \\ ORCID \\ Katarína Ražná: https://orcid.org/0000-0003-2121-131X
}

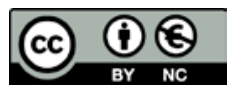

Article Details:

Received: 2021-07-11

Accepted: 2021-08-27

Available online: 2021-11-30

DOI: https://doi.org/10.15414/ainhlq.2021.0025

\begin{abstract}
Ginkgo biloba L. is the oldest species of tree on our planet. It is a dioecious species characterized by exceptional resistance to climate change and natural influences, which predetermines it as a suitable species for urban planting. Not to be overlooked is the ginkgo benefit in terms of the medical effects of leaf metabolites. Due to its aesthetic value ginkgo is becoming popular in family gardens. The aim of our research was the evidence and description of ginkgo trees in Slovakia. The study presents the completion of data on the cultural distribution of this tree. We confirmed the occurrence on more than one hundred localities (103 localities). Some previously registered localities (18) were not found, or trees were felled for various reasons (5). We evaluated the basic dendrometric and growth parameters of the oldest ginkgo trees (aged 242 - 111 years), found in 35 localities of Slovakia. The presented research results bring several new information concerning the tree gender determination, tree habitus, phenological rhythm of development and others. Morphologically interesting solitary trees, including trees with shoots known as lignotubers or "basal chichi" (locality Hajná Nová Ves), or the occurrence of fruits on the leaves, referred to as cv. Ohatsuki (Lučenec) are described and documented by photos.
\end{abstract}

Keywords: maidenhair tree, locality, occurrence, Slovak Republic

\section{Introduction}

Ginkgo biloba L. is a dioecious species endemic to China (Li et al., 2009). It is a famous living fossil and is the only known extant representative of Ginkgophyta (He et al., 2015; Šmarda et al., 2016). It contains several different biologically active compounds which play a role in defense mechanisms against insects, bacteria and fungi (Singh et al., 2008). The leaves of ginkgo are a rich source of compounds with antioxidant activity (van Beek, 2000) and are commonly used as phytomedicines in the treatment of atherosclerosis and cerebrovascular insufficiency (Kleijnen and Knipschild,
1992; Xie et al., 2003) depression, memory loss, headaches, and vertigo (Diamont et al., 2000). A recent study of extracts from the leaves of Ginkgo biloba L. from some Slovakian localities showed that these extracts can be used as antimicrobial and antioxidant additives due to their significant antioxidant and antimicrobial activity which was sample-specific (Ražná et al., 2020).

In Slovakia, Ginkgo biloba L. is relatively little known, although in historical parks and gardens it grows in several places, from plantings from the late $18^{\text {th }}$ and $19^{\text {th }}$ centuries (e. g. Bratislava, Topolčianky, Nová Ves nad Žitavou, Beladice, Lučenec, Betliar, Jasov, Košice,

\footnotetext{
*Corresponding Author: Katarína Ražná, Slovak University of Agriculture in Nitra, Faculty of Agrobiology and Food Resources, Institute of Plant and Environmental Sciences, Trieda Andreja Hlinku 2, 949 76, Nitra, Slovakia $\triangle$ katarina.razna@uniag.sk
} 
Humenné, etc.) (Tokár, 1968; Tokár, 1970; Ražná et al., 2014). Current horticultural practice confirms that ginkgo belongs to a small group of species capable of resisting urban exhaustion, salinization, and water scarcity (Raček et al., 2009, 2010, 2011), and at the same time, with its aesthetic value, effectively beautifies the urban environment. From the horticultural point of view, ginkgo is becoming more and more popular, and its coloured and shaped cultivars are gaining more and more space in family gardens (Tomaško, 2004; Hrubík et al., 2011).

We began research into the cultural distribution and occurrence of Ginkgo biloba L. in 2007. We focused first on the districts of Nitra and Zlaté Moravce, and later the entire Nitra self-governing region. The most suitable conditions for research were provided by fruit trees in the State Forest Park in Topol'čianky, the Arborétum Mlyňany of the Slovak Academy of Sciences, historical parks in Nová Ves nad Žitavou, Beladice, and trees planted in the Nitra City Park in Sihot'. Later, we extended the research to the districts of Topol'čany, Trnava, Levice, Nové Zámky and Komárno. Intensive field research continued in 2010-2011 in other districts of Slovakia (former districts of the West and Central Slovakia regions). We presented a summary of the acquired knowledge and more comprehensive research in two monographs (Ražná et al., 2014; Ražná and Hrubík, 2016). The mentioned publications contain a comprehensive overview of the determined taxation values, complete biological data, horticultural values, and an evaluation of the health status of ginkgo trees that grow in Slovakia. The publication also includes a complete list of trees growing in historical parks, street plantings (mostly as tree lines and alley plantings), and other objects of public (and accessible private) greenery in towns and villages in Slovakia. In addition to the results of field research, considerable attention was paid to a genomic screening of polymorphism using molecular markers, as well as antioxidant and antimicrobial parameters of selected individuals of ginkgo (Ražná et al., 2019; Ražná et al., 2020).

The impetus for concluding and completing the research on the cultural distribution of Ginkgo biloba in Slovakia was, on the one hand, the processing of scientific knowledge acquired so far, but especially the commitment to conclude current and interesting issues of spreading one of the rare cultural trees Ginkgo biloba in Slovakia. The first findings on the distribution of ginkgo in Slovakia were obtained as early as 1965-1975, as part of cooperation on extensive and comprehensive research in the Arborétum Mlyňany - Institute of Dendrobiology of the
Slovak Academy of Sciences. When selecting research sites, we therefore, accepted the published results of the cultural distribution of foreign trees in Slovakia (including Ginkgo biloba), (Benčat', 1982). It contains a total of 80 sites ( 75 historic parks, 5 other greenery). From the available literature, internet sources, other sources, as well as from our own findings, we supplemented and evaluated the ginkgo trees in other (new) localities. For the purposes of this publication, we selected a list of the oldest trees of Ginkgo biloba, growing in Slovakia. The locality contains the name and registration number of the historical park and garden (P - 44), district - according to the current territorial division of the territory (TO - Topol'čany), period of the foundation of the dendrological building in the relevant century (e. g. 19/1 - first half of the $19^{\text {th }}$ century, with the designation of the years - 1850). For this purpose, we selected several figures of interesting solitary trees and conclusions based on our long-term research, concerning tree habitus and gender differentiation features.

\section{Material and methodology}

\section{Biological material}

In research of cultural extension of Ginkgo biloba, we followed methodologies and working procedures. As already mentioned, we have fully accepted the well-known and published list of identified localities (Benčat', 1982) (Figure 1), including other, new localities identified from other unpublished, internet sources, as well as information on previously registered or otherwise registered trees (e. g. stateprotected trees, where old and memorial ginkgo trees were included). At each locality, which we personally evaluated, we surveyed the existing ginkgo trees, on which we obtained basic taxation data (trunk circumference, trunk diameter $1.3 \mathrm{~m}$ above the ground; tree height, crown width, health status, horticultural value - according to the 5-point scale, in the case of larger trees, we also determined the circumference of the trunk at the ground) (Hrubík et al., 2011) and made an up - to - date photo documentation of the trees at a specific locality.

When determining the main dendrometric dimensions of ginkgo trees, we used a specialized textile band, which measures, on one side of the band, in units of $\mathrm{cm}$, and on the other side of the band, are measured units converted to the values of the average. During the field research, we used this textile band to measure the circumference of the tree trunk, thus also obtaining the measured value of the trunk diameter. We measured 


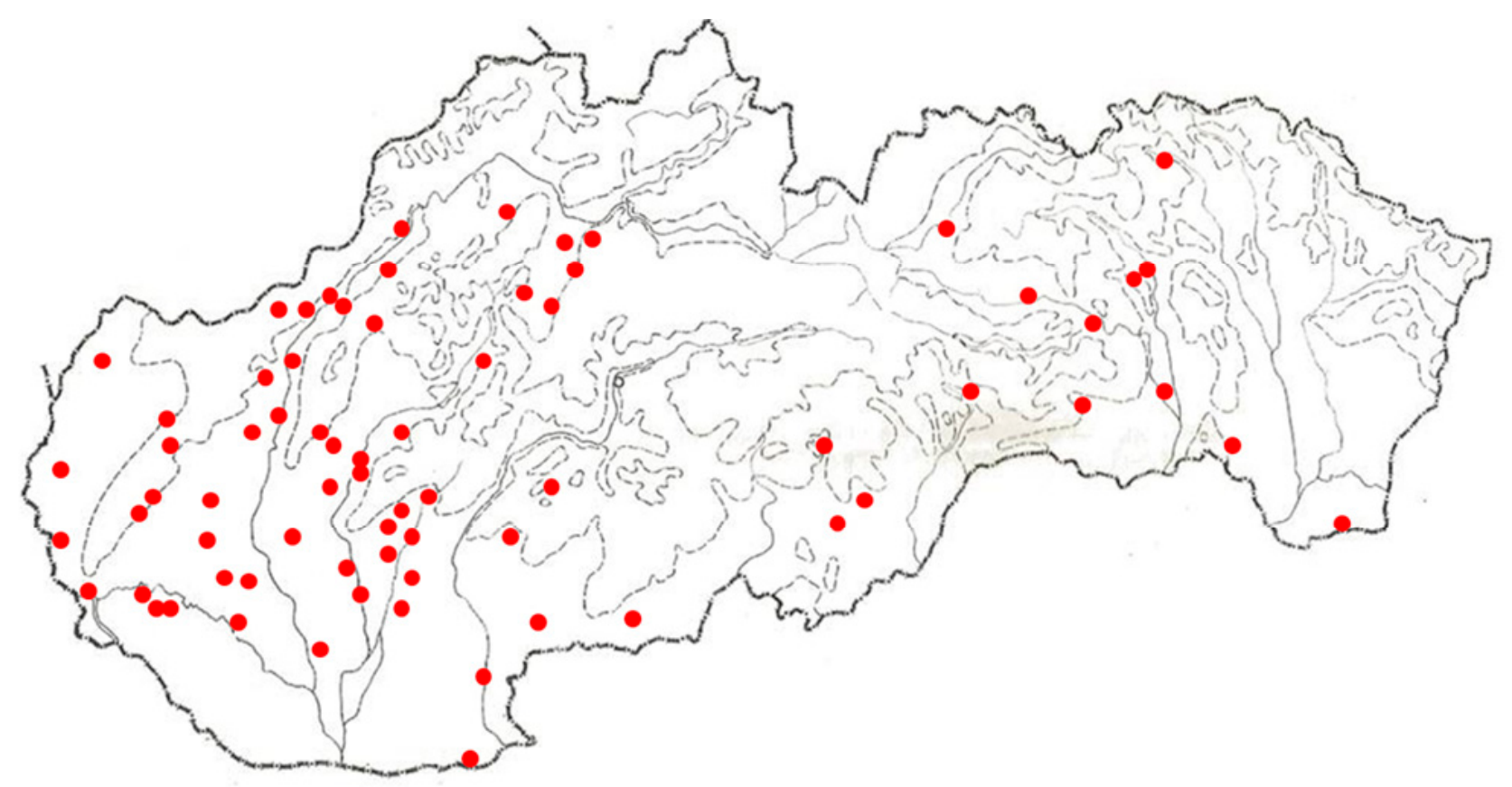

Figure 1 Localities of occurrence of Ginkgo biloba L. identified by Benčat' (1982)

the height of the tree with a SUUNTO altimeter; we estimated the width of the crown by a step in two intersecting directions according to the shape of the crown (however, most of them were solitary trees with a regular crown). We identified the sex of the trees. The blossoms are dioecious, male and female blossoms occurring separately on two different trees. There are some additional tree gender differentiation features as crown habitual marks, and the angle of protruding lateral branches.

Determining the age of Ginkgo biloba L. trees was, in our experience, the most problematic. For some trees, especially younger (juvenile) individuals, the data of the tree age or year of planting are relatively accurate (a value must be added to this data, it is 5-10 years from the cultivation of seedlings from seed in the nursery until the planting of seedlings in a specific location). In addition, we used the Pressler drill (number of annual rings on a dendrometric borehole) to determine the age, but the evaluation of the obtained results was time and money consuming (we obtained a total of 25 boreholes), so we did not continue in this approach. However, we used a mathematical formula $\mathrm{V}=(5 /[\pi \times \mathrm{RL}]) \times \mathrm{d}$, where $\mathrm{d}$ is the diameter of the trunk (in $\mathrm{cm}$ ) and RL is the width of the annual ring (in cm) (Kolařík, 2005).

Another way of determining the age of trees works with information about the period of origin, respectively the establishmentofa specifichistoricalpark(dendrological building), the most often it is the $18^{\text {th }}$ and $19^{\text {th }}$ to $20^{\text {th }}$ centuries $(1901-2000)$. Determining the age of trees according to the circumference of the trunk ( $d 1.3 \mathrm{~m}$ ) is another option, but the most accurate is the year of planting of a particular tree.

\section{Research area}

All obtained data on individual trees were processed in a tabular overview by locality, including data on a specific historical park or object of other greenery. The tabular overview includes findings on the tree gender (male - $\mathrm{m}$; and female - f), juvenile age (until the period of occurrence of seeds, or male and female generative organs), and the occurrence of seed propagation (seedlings under fruiting female trees). For completeness, we present a table of localities of cultural extension of Ginkgo biloba in Slovakia according to Benčat' (1982).

\section{Description of a model tree, Ginkgo biloba L.}

Ginkgo is a long-lived deciduous tree. It can reach more than a thousand years old. Depending on the growing conditions, mature trees reach a height from 20 to $40 \mathrm{~m}$. The crown is somewhat ovoid to obovoid, tending to be asymmetric, primary branches ascending at ca. $45^{\circ}$ from trunk (Flora of North America). In males' trees, it is usually slimmer, in females' ones bushier (Figure 2). Ginkgo trees produce two types of shoots: long shoots with widely spaced leaves that subtend axillary buds; and short shoots with clustered leaves that lack both internodes and axillary buds. Under stressful growing 
Table 1 Localities of cultural extension of Ginkgo biloba L. in Slovakia according to Benčat’ (1982)

\begin{tabular}{|c|c|c|}
\hline District & Localities & Registration number of localities \\
\hline Bardejov & Bardejov spa & P-328 \\
\hline \multirow[t]{3}{*}{ Bratislava-city } & Bratislava & P-103, $105,108,110$ \\
\hline & Častá - Červený Kameň & $\mathrm{P}-424$ \\
\hline & Malacky & SG \\
\hline \multirow[t]{4}{*}{ Bratislava-city surroundings } & Modra & P-90 \\
\hline & Stupava & P-96 \\
\hline & Tomášov & P-100 \\
\hline & Hubice & P-192 \\
\hline \multirow[t]{2}{*}{ Dunajská Streda } & Sládkovičovo & $\mathrm{P}-145$ \\
\hline & Tomášikovo & P-148 \\
\hline Komárno & Kravany nad Dunajom & P-189 \\
\hline \multirow{3}{*}{ Košice } & Jasov & P-396 \\
\hline & Košice & P-382, 388, 390, 392 \\
\hline & Bohunice & P-149 \\
\hline \multirow[t]{2}{*}{ Levice } & Horné Semerovce & P-158 \\
\hline & Pohronský Ruskov & SG \\
\hline \multirow{2}{*}{ Lučenec } & Lučenec - Ipel’ské brickfield & SG \\
\hline & Nenince & P-276 \\
\hline \multirow{4}{*}{ Martin } & Abramová & SG \\
\hline & Martin & P-228 \\
\hline & Mošovce & $\mathrm{P}-231$ \\
\hline & Necpaly & $\mathrm{P}-230$ \\
\hline \multirow{10}{*}{ Nitra } & Turčianska Štiavnička & P-225 \\
\hline & Báb & P-133 \\
\hline & Beladice & P-124 \\
\hline & Ivanka pri Nitre & SG \\
\hline & Mlyňany Arborétum & P-129 \\
\hline & Nová Ves nad Žitavou & P-135 \\
\hline & Sl'ažany & P-118 \\
\hline & Šurianky & P-114 \\
\hline & Telince & SG \\
\hline & Topol'čianky & P-115 \\
\hline \multirow{4}{*}{ Nové Zámky } & Komjatice & P-166 \\
\hline & Obid & SG \\
\hline & Palárikovo & P-174 \\
\hline & Trávnica & P-169 \\
\hline Poprad & Kežmarok & SG \\
\hline \multirow{2}{*}{ Považská Bystrica } & Klobušice & P-217 \\
\hline & Horenická Hôrka - Medné & P-205 \\
\hline \multirow{2}{*}{ Prešov } & Malý Šariš & P-348 \\
\hline & Župčany & P-347 \\
\hline \multirow{2}{*}{ Prievidza } & Prievidza & SG \\
\hline & Bojnice & park by the castle \\
\hline
\end{tabular}


Table 1 Localities of cultural extension of Ginkgo biloba L. in Slovakia according to Benčat' (1982)

\begin{tabular}{|c|c|c|}
\hline District & Localities & Registration number of localities \\
\hline \multirow{3}{*}{ Rimavská Sobota } & Hnúšta & SG \\
\hline & Rimavská Sobota & P-292 \\
\hline & Vel'ký Blh & P-286 \\
\hline Rožňava & Betliar & P-407 \\
\hline \multirow{2}{*}{ Senica nad Myjavou } & Cerová-Lieskové & P-30 \\
\hline & Gbely & P-28 \\
\hline \multirow{2}{*}{ Spišská Nová Ves } & Bystrany & P-357 \\
\hline & Jaklovce & P-359 \\
\hline \multirow{5}{*}{ Topol'čany } & Hajná Nová Ves & P-44 \\
\hline & Horné Obdokovce & $\mathrm{P}-50$ \\
\hline & Janova Ves & $\mathrm{P}-42$ \\
\hline & Oponice & $\mathrm{P}-52$ \\
\hline & Kovarce & $\mathrm{P}-48$ \\
\hline \multirow{2}{*}{ Trebišov } & Kazimír & P-416 \\
\hline & Pribeník & P-418 \\
\hline \multirow{7}{*}{ Trenčín } & Adamovské Kochanovce & P-8 \\
\hline & Částkovce & $\mathrm{P}-21$ \\
\hline & Kočovce & $\mathrm{P}-17$ \\
\hline & Motešice & $\mathrm{P}-13$ \\
\hline & Trenčín & P-7, SG \\
\hline & Záblatie & P-4 \\
\hline & Zemianske Podhradie & $\mathrm{P}-12$ \\
\hline \multirow{5}{*}{ Trnava } & Piešttany & P-58 \\
\hline & Rakovice & P-66 \\
\hline & Smolenice & P-70 \\
\hline & Trnava & P-78, SG \\
\hline & Voderady & P-84 \\
\hline Žiar nad Hronom & Banská Štiavnica & P-246 \\
\hline Žilina & Rajecké Teplice & P-223 \\
\hline
\end{tabular}

Note: SG - surrounding greenery, that is, the tree is in a territory other than a historic park or garden

conditions, Ginkgo can produce secondary trunks at or just below ground level. These secondary stems originate from root-like, positively geotropic shoots known as lignotubers or "basal chichi" (van Beek, 2000) (Figure 3). The bark is smooth, gray, relatively quickly turns into a brown-gray bark, which is cracked in longitudinal irregular plates (Figure 4c).

Buds are brown, globose, scales imbricate, margins scarious (Flora of North America) (Figure 4e). Leaves are 30-60 × 40-100 mm fan-shaped (with long stalks) with dichotomously branched veins (Figure 4a). They are flat, firm, leathery, light green, golden yellow in autumn before falling (Figure 4d). It blooms in May (Figure $4 \mathrm{~b}$ ). The flowers are dioecious, growing only on shortened shoots (Pagan and Randuška. 1988). Microsporophylls occur in small, conelike clusters (are pendulous). In females' trees, an upright $25-40 \mathrm{~mm}$ long stalk grows in the armpits of scales or leaves, which is widened at the end. Ovules occur in pairs at the ends of a short stalk. The ovule is surrounded by a cup-shaped cushion called a collar. Seeds obovoid to ellipsoid, yellow to orange, $2.3-2.7 \times 1.9-2.3 \mathrm{~cm}$, mostly 1.1-1.2 times longer than broad, glaucous, rugose, with an apical scar, maturing in a single season, usually 1 per peduncle, occasionally polyembryonic, outer coat foul-smelling; peduncles orange, glaucous, ridged, 3-9.5 cm, collar broadly elliptic, $7.2-8.6 \mathrm{~mm}$ broad (Vreštiak and Osvald, 1994; Klečková, 2010; 


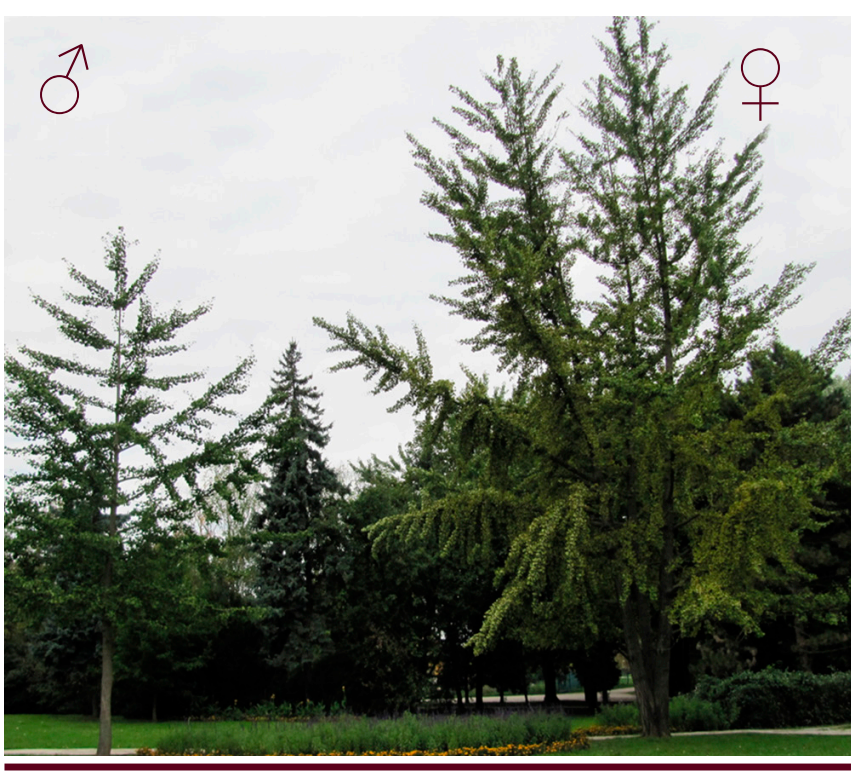

Figure 2 Male (left) and female (right) tree of ginkgo grown in city park in Nitra Photo by Pavel Hrubík

Zvolen (3 pregenerative individual trees). In other localities we recorded from 1 to 2 trees. The total number of localities (towns and villages) with the occurrence of Ginkgo biloba was 103. In addition, we

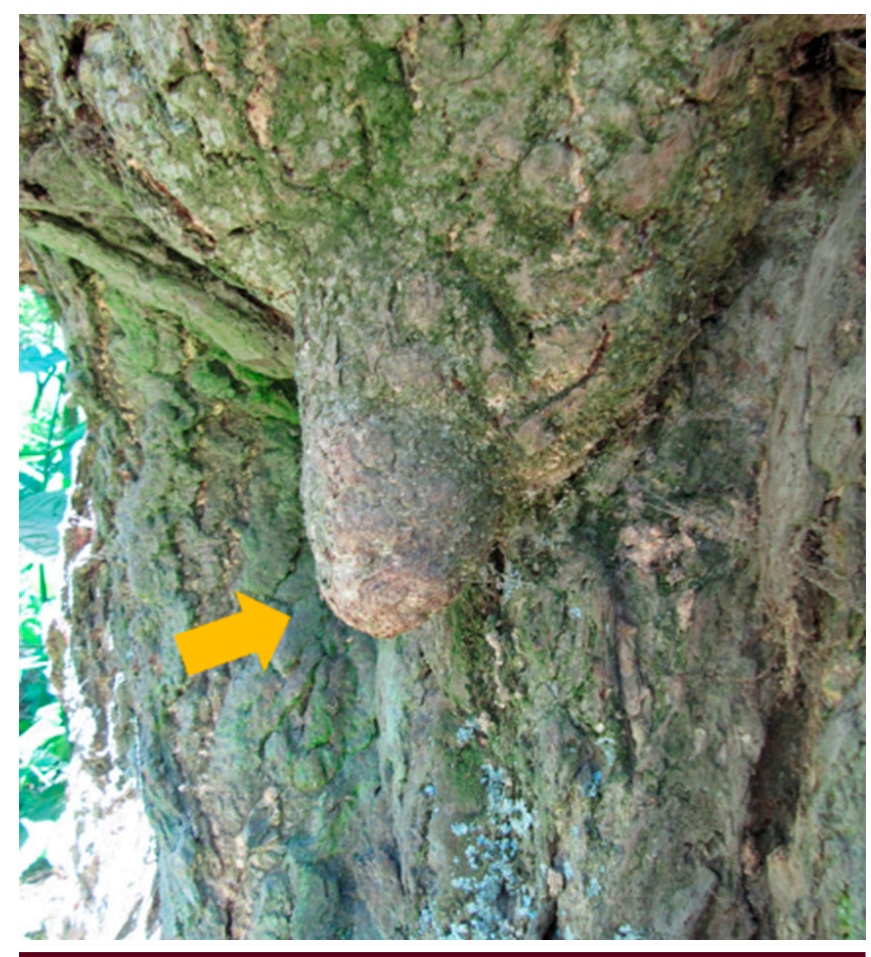

Figure 3 Shoots known as lignotubers or "basal chichi". Ginkgo grows in historical park near the manor house, Hajná Nová Ves Photo by Pavel Hrubík
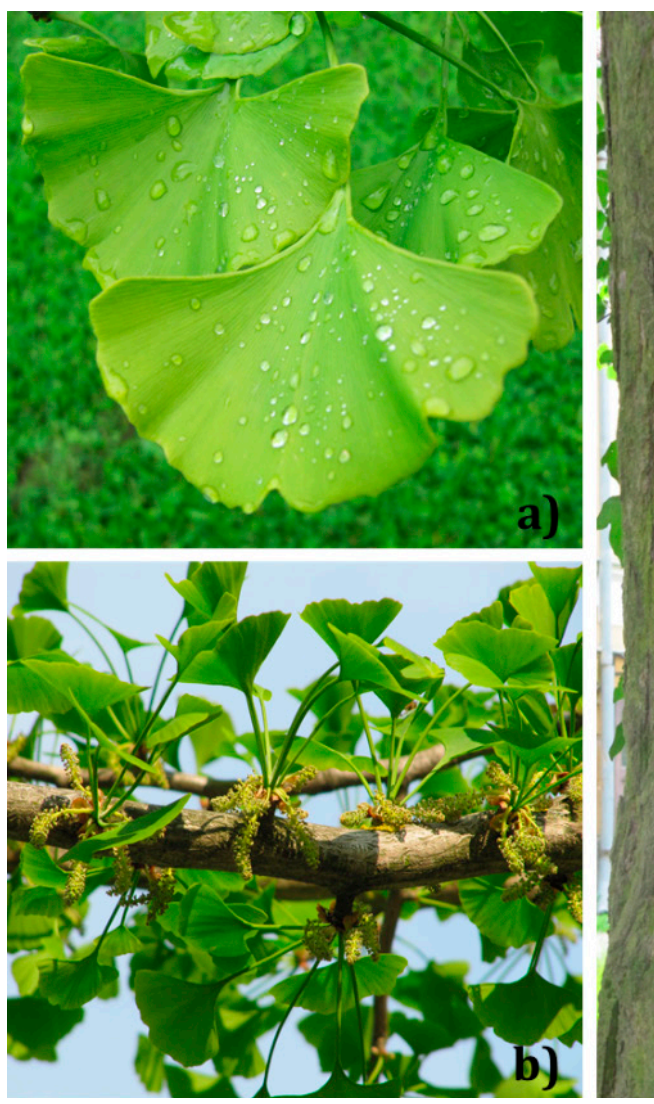

Figure 4 Fan-shaped leaves with dichotomously branched veins (a), scaly stamens in short spikes on male tree (b), bark (c),

$\begin{array}{ll}\text { Figure } 4 & \begin{array}{l}\text { Fan-shaped leaves with dichotomously branch } \\ \text { golden-yellow leaves in autumn (d), buds (e) }\end{array}\end{array}$ Photos by Pavel Hrubík

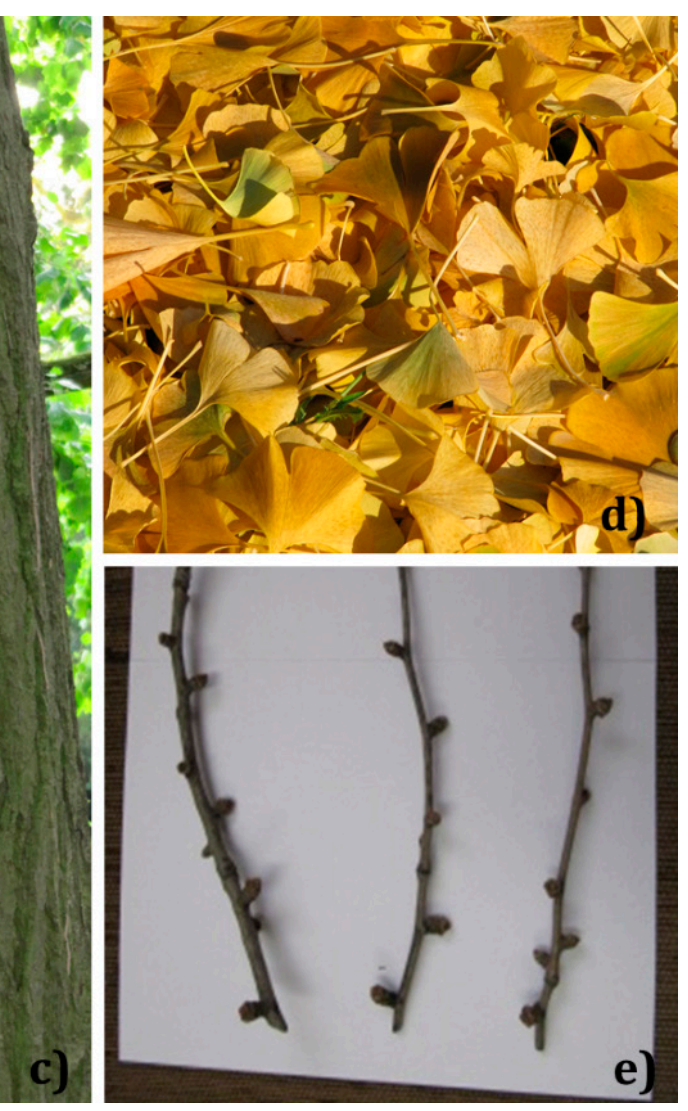

)

Phos bavel Hrubt 

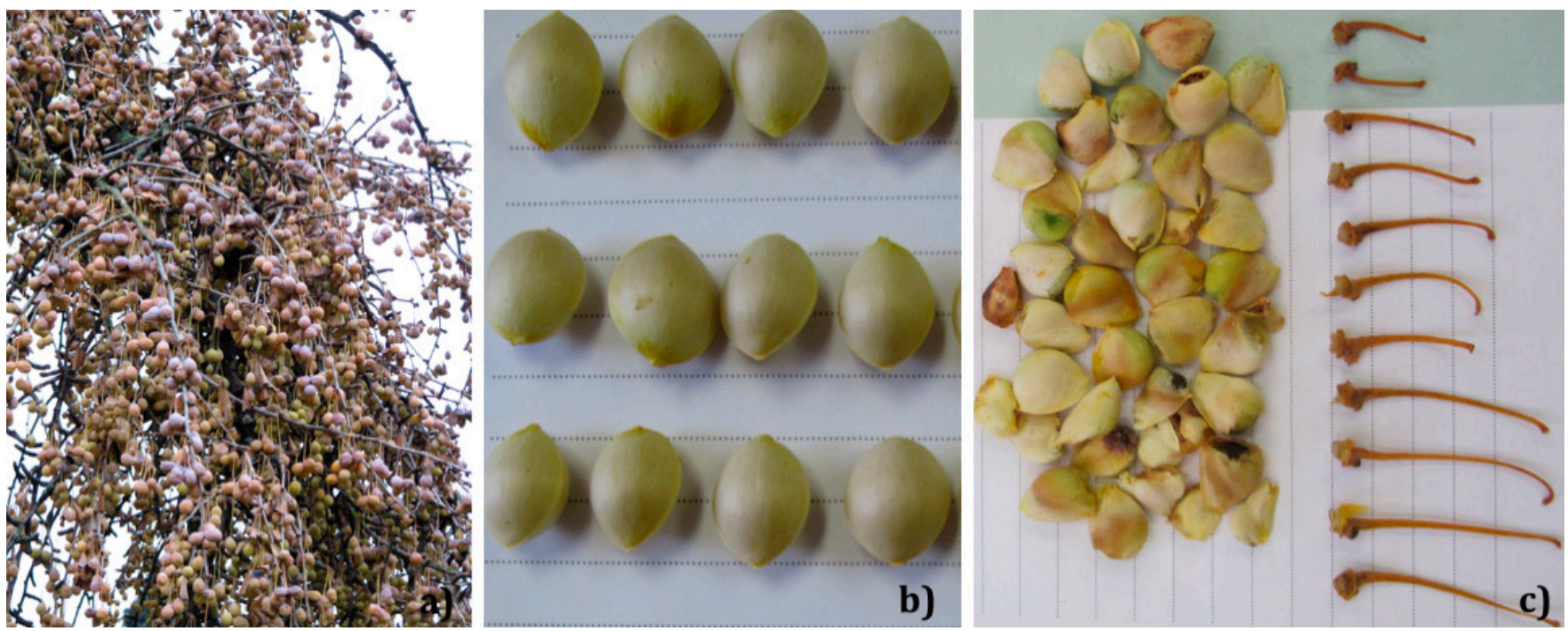

Figure 5 Fruits on female tree (a), seeds (b) and seed of irregular shape with stalks

Photos by Pavel Hrubík

Flora of North America). Figure 5a shows fruits on the female tree, seeds after removal of the flesh (Figure 5b), and irregular shape of seeds from the ginkgo grown in Lučenec.

Ginkgo seeds are dormant when they fall from the tree because the embryo is not fully developed, being only about 4 to $5 \mathrm{~mm}$ in length. If seeds are collected shortly after dispersal, are cleaned, and placed in a warm greenhouse, the embryo will grow to its full size, 10 to $12 \mathrm{~mm}$ in length and germinate within eight to ten weeks. Ginkgo shows a long juvenile period, typically not reaching sexual maturity until 20 to 30 years of age (van Beek, 2000). The foul odour associated with mature seeds is result of the presence of butanoic and hexanoic acids (Zhou and Wang, 2020).

\section{Results and discussion}

\section{Main dendrometric values of ginkgo trees}

In Slovakia, we found the occurrence and cultural distribution of Ginkgo biloba in 103 localities (Table 2). The total number of trees reached 292 individuals (including new plantings in Bratislava - Košická street, tree line of 29 trees in the middle dividing grass strip, Ginkgo biloba cv. Fastigiata; group plantings at the level crossing with Bajkalská street, 30 pieces; in Senica 25 trees in a two-sided alley on Hurbanova street, of which 4 in the tree line on Hviezdoslavova street, in Žilina above the shopping center, Ginkgo biloba (cv. Fastigiata - 5 pregenerative individuals). Some Ginkgo biloba trees were felled (despite protests from the civil public) at the site of the programmed construction of a hotel in Trenčín (one male tree in 2008); for hygienic and safety reasons (Košice, Rázusova street; Rimavská Sobota, Hviezdoslavova street no. 25); technical damage to the adjacent building (Košice, campus of the University of Veterinary Medicine and Pharmacy); tree damaged and destroyed during lawn mowing (Kežmarok, Gymnázium P.O. Hviezdoslava); a total of 5 Ginkgo trees.

The most numerous localities (apart from the already mentioned pregenerative individuals in tree lines and alley plantings) were recorded in the Mlynnany Arborétum SAS, 17 trees (of which one is a male tree, three females, fruiting trees, 13 pregenerative trees); In Bratislava, 14 trees (of which 5 in the Botanical Garden of the Charles University in Prague, 2 in Petržalka, Janko Král' Park; 2 on Dunajská Street in the courtyard of the fitness centre), the tree line on Košická Street is mentioned separately; 9 trees in Košice (of which 5 trees in the premises of the L. Pasteur University Hospital; 7 trees in Pieštany (3 Parks on the Island, 4 Parks at the Secondary Vocational Horticultural School), 5 trees in Palárikovo ( 3 males and 2 females trees, 3 trees in Nitra (of which 2 trees - male and female tree, planted in 1963 in the Nitra City Park in Sihot'); more cultivars and pregenerative individuals are still growing in the Botanical Garden of the Slovak University of Agriculture, which were not included in the research. In Topolčianky we register 4 trees (of which one male and 3 female trees); in Nová Ves nad Žitavou 4 trees (female trees); we recorded 3 trees in the following localities: Bojnice (male individuals); Topolčany (one female tree, 2 pregenerative individuals); Trenčín ( 2 female and 1 male tree); Trnava ( 2 male, 1 female tree); Banská Štiavnica (2 male and 1 female tree); 
Table 2 Characteristics of Ginkgo biloba L. trees in Slovakia. The data come from the research period in 2014-2016

\begin{tabular}{|c|c|c|c|c|c|c|}
\hline Locality & $\begin{array}{c}\text { Trunk } \\
\text { circumference }(\mathrm{cm})\end{array}$ & $\begin{array}{c}\text { Trunk } \\
\text { diameter }(\mathrm{cm}) \\
\end{array}$ & $\begin{array}{c}\text { Tree height } \\
\text { (m) }\end{array}$ & $\begin{array}{c}\text { Crown } \\
\text { width }(\mathrm{m})\end{array}$ & $\begin{array}{c}\text { Gender } \\
(\hat{0},+)\end{array}$ & Age \\
\hline Abramová (SG), TR & 238 & 76 & 14 & $18 \times 14$ & 우 & 121 \\
\hline $\begin{array}{l}\text { Arborétum Mlyňany SAV, } \\
\text { (P-129), 1892, ZM (11) }\end{array}$ & 92 & 29,3 & 16 & $10 \times 4$ & $\hat{0}$ & 47 \\
\hline $\begin{array}{l}\text { Arborétum Mlyňany SAV, } \\
\text { (P-129), 1964, ZM (1) }\end{array}$ & 30 & 9,6 & 10 & $3 \times 3$ & juv. & 15 \\
\hline $\begin{array}{l}\text { Arborétum Mlyňany SAV, } \\
\text { (P-129), 1964, ZM (10) }\end{array}$ & 18 & 6,0 & 7 & $3 \times 3$ & ㅇ & 10 \\
\hline $\begin{array}{l}\text { Arborétum Mlyňany SAV, } \\
\text { (P-129), 1964, ZM (12) }\end{array}$ & 60 & 19,2 & 11 & $5 \times 6$ & juv. & 31 \\
\hline $\begin{array}{l}\text { Arborétum Mlyňany SAV, } \\
\text { (P-129), 1964, ZM (13) }\end{array}$ & 88 & 28,2 & 14 & $8 \times 8$ & juv. & 38 \\
\hline $\begin{array}{l}\text { Arborétum Mlyňany SAV, } \\
\text { (P-129), 1964, ZM (14) }\end{array}$ & 90 & 28,8 & 16 & $8 \times 8$ & juv. & 44 \\
\hline $\begin{array}{l}\text { Arborétum Mlyňany SAV, } \\
\text { (P-129), 1964, ZM (15) }\end{array}$ & 53 & 16,9 & 14 & $4 \times 4$ & juv. & 26 \\
\hline $\begin{array}{l}\text { Arborétum Mlyňany SAV, } \\
\text { (P-129), 1964, ZM (16) }\end{array}$ & 43 & 13,7 & 14 & $3 \times 3$ & juv. & 22 \\
\hline $\begin{array}{l}\text { Arborétum Mlyňany SAV, } \\
\text { (P-129), 1964, ZM (2) }\end{array}$ & 17 & 5,4 & 6 & $3 \times 3$ & juv. & 12 \\
\hline $\begin{array}{l}\text { Arborétum Mlyňany SAV, } \\
\text { (P-129), 1964, ZM (3) }\end{array}$ & 23 & 7,4 & 8 & $3 \times 3$ & juv. & 9 \\
\hline $\begin{array}{l}\text { Arborétum Mlyňany SAV, } \\
\text { (P-129), 1964, ZM (4) }\end{array}$ & 82 & 26,2 & 16 & $6 \times 6$ & 우 & 41 \\
\hline $\begin{array}{l}\text { Arborétum Mlyňany SAV, } \\
\text { (P-129), 1964, ZM (5) }\end{array}$ & 31 & 9,9 & 8 & $5 \times 6$ & juv. & 16 \\
\hline $\begin{array}{l}\text { Arborétum Mlyňany SAV, } \\
\text { (P-129), 1964, ZM (6) }\end{array}$ & 64 & 20,4 & 10 & $5 \times 5$ & juv. & 32 \\
\hline $\begin{array}{l}\text { Arborétum Mlyňany SAV, } \\
\text { (P-129), 1964, ZM (8) }\end{array}$ & 66 & 20,7 & 12 & $6 \times 8$ & juv. & 33 \\
\hline $\begin{array}{l}\text { Arborétum Mlyňany SAV, } \\
\text { (P-129), 1964, ZM (9) }\end{array}$ & 70 & 22,4 & 12 & $8 \times 10$ & q & 36 \\
\hline $\begin{array}{l}\text { Arborétum Mlyňany SAV, } \\
\text { (P-129), KD, ZM }\end{array}$ & 18 & 5,8 & 5 & $2 \times 2$ & juv. & 9 \\
\hline $\begin{array}{l}\text { Arborétum Mlyňany SAV, } \\
\text { (P-129), 1964. ZM (17) }\end{array}$ & 49 & 15,7 & 12 & $3 \times 3$ & juv. & 24 \\
\hline Báb (P-133), NR & 187 & 59,7 & 16 & $10 \times 12$ & $\hat{0}$ & 95 \\
\hline $\begin{array}{l}\text { Bánovce nad Bebravou, } \\
\text { City park, BN }\end{array}$ & 101 & 32,3 & 16 & $12 \times 6$ & 0 & 51 \\
\hline $\begin{array}{l}\text { Banská Štiavnica (P-246), } \\
\text { Botanical garden, ZH }\end{array}$ & 178 & 56,8 & 20 & $3 \times 8$ & ㅇ & 90 \\
\hline $\begin{array}{l}\text { Banská Štiavnica (P-246), } \\
\text { Botanical garden, ZH }\end{array}$ & 191 & 69,9 & 18 & $12 \times 10$ & $\widehat{0}$ & 111 \\
\hline $\begin{array}{l}\text { Banská Štiavnica (P-246), } \\
\text { Botanical garden, ZH }\end{array}$ & 112 & 36,0 & 16 & $8 \times 10$ & $\widehat{0}$ & 57 \\
\hline Beladice (P-124), ZM & 259 & 82,5 & 18 & $16 \times 16$ & $0^{\pi}$ & 131 \\
\hline Beladice (P-124), ZM & 237 & 75,2 & 20 & $16 \times 16$ & q & 119 \\
\hline Betliar (P-407), RV & 233 & 74,3 & 19 & $19 \times 14$ & 0 & 118 \\
\hline Betliar (P-407), RV & 12 & 4,0 & 3 & $1,5 \times 1,5$ & juv. & 6 \\
\hline
\end{tabular}


First continuation of table 1

\begin{tabular}{|c|c|c|c|c|c|c|}
\hline Locality & $\begin{array}{c}\text { Trunk } \\
\text { circumference }(\mathrm{cm})\end{array}$ & $\begin{array}{c}\text { Trunk } \\
\text { diameter }(\mathrm{cm}) \\
\end{array}$ & $\begin{array}{l}\text { Tree height } \\
\text { (m) }\end{array}$ & $\begin{array}{c}\text { Crown } \\
\text { width }(\mathrm{m})\end{array}$ & $\begin{array}{c}\text { Gender } \\
(\hat{0},+)\end{array}$ & Age \\
\hline Bojnice (P-1, 2, 3), PD & 276 & 87,9 & 21 & $10 \times 10$ & $\pi$ & 140 \\
\hline Bojnice (P-1, 2, 3), PD & 218 & 69,5 & 16 & $10 \times 10$ & 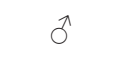 & 110 \\
\hline Bojnice (P-1, 2, 3), PD, $415 \mathrm{~cm}$ & 290 & 92,4 & 26 & $12 \times 12$ & $\hat{\sigma}$ & 147 \\
\hline $\begin{array}{l}\text { Bratislava (P-103), } \\
\text { Botanical garden UK, BA }\end{array}$ & 168 & 53,5 & 14 & $15 \times 15$ & q & 85 \\
\hline $\begin{array}{l}\text { Bratislava (P-103), } \\
\text { Botanical garden UK, BA }\end{array}$ & 222 & 70,7 & 16 & $8 \times 8$ & 우 & 112 \\
\hline $\begin{array}{l}\text { Bratislava (P-103), } \\
\text { Botanical garden UK, BA }\end{array}$ & 141 & 45,0 & 14 & $14 \times 14$ & q & 72 \\
\hline $\begin{array}{l}\text { Bratislava (P-103), } \\
\text { Botanical garden UK, BA }\end{array}$ & 125 & 37,0 & 12 & $6 \times 5$ & q & 58 \\
\hline $\begin{array}{l}\text { Bratislava (P-103), } \\
\text { Botanical garden UK, BA }\end{array}$ & 118 & 37,2 & 17 & $15 \times 15$ & q & 53 \\
\hline Bratislava (P-105), Mountain park, BA & 42 & 13,4 & 8 & $5 \times 3$ & juv. & 21 \\
\hline $\begin{array}{l}\text { Bratislava (P-108), PKO, statue of D. } \\
\text { Jurkoviča, BA }\end{array}$ & 105 & 33,5 & 14 & $8 \times 8$ & $\hat{0}$ & 53 \\
\hline $\begin{array}{l}\text { Bratislava, } \\
\text { Bohúňova St. 4, BA }\end{array}$ & 114 & 36,4 & 18 & $6 \times 6$ & q & 58 \\
\hline $\begin{array}{l}\text { Bratislava, } \\
\text { Dunajská St., BA, } 168 \mathrm{~cm}\end{array}$ & 124 & 39,5 & 14 & $7 \times 6$ & q & 63 \\
\hline $\begin{array}{l}\text { Bratislava, Dunajská St., BA, park near } \\
\text { FITNES }\end{array}$ & 60 & 19,0 & 8 & $4 \times 3$ & $\hat{0}$ & 30 \\
\hline Bratislava, Godrova St. 8, BA & 215 & 65,0 & 16 & $12 \times 12$ & q & 103 \\
\hline $\begin{array}{l}\text { Bratislava, Košická St., alley, BA, 2008, } \\
29 \text { pcs. }\end{array}$ & $18-35$ & $5,5-11,0$ & $4-5$ & $2 \times 2$ & juv. & 18 \\
\hline Bratislava, At the Habán mill, BA & 29 & 9,3 & 6 & $2 \times 2$ & juv. & 15 \\
\hline $\begin{array}{l}\text { Bratislava, J. Král' Gardens, } \\
\text { 18/2-1751, BA }\end{array}$ & 165 & 52,5 & 16 & $14 \times 13$ & q & 83 \\
\hline Bratislava, J. Král' Gardens, BA, $540 \mathrm{~cm}$ & 467 & 148,8 & 20 & $28 \times 24$ & q & 236 \\
\hline $\begin{array}{l}\text { Bratislava, Slovanet Ltd., } \\
\text { group planting. Bajkal St. }\end{array}$ & $17-21(30 \mathrm{ks})$ & $5-7$ & $3-5-6$ & $1 \times 2$ & juv. & 10 \\
\hline Brezová pod Bradlom, PS, SE & 168 & 53,6 & 16 & $15 \times 15$ & 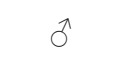 & 65 \\
\hline Brusno Spa (P-242) & 9 & 2,9 & 3,5 & $1 \times 2$ & juv. & 10 \\
\hline Budimír, at the chapel, (P-378), KE & 155 & 49,4 & 22 & $7 \times 7$ & 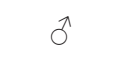 & 79 \\
\hline Bystrany (P-357), SN & 179 & 57,0 & 22 & $8 \times 8$ & q & 91 \\
\hline Bystrany (P-357), SN & 57 & 18,2 & 3 & $3 \times 3$ & q & 29 \\
\hline Cerová-Lieskové (P-30), SE & 183 & 58,3 & 26 & $12 \times 14$ & $\hat{\sigma}$ & 93 \\
\hline $\begin{array}{l}\text { Častá (Červený Kameň), } \\
\text { PK, 447/142,4 cm }\end{array}$ & 375 & 122,2 & 14 & $12 \times 14$ & q & 194 \\
\hline Častkovce (P-21), NM & 172 & 54,5 & 19 & $12 \times 8$ & ㅇ & 87 \\
\hline Červený Hrádok, ZM, 1964 & 26 & 8,3 & 9 & $2 \times 2$ & juv. & 13 \\
\hline Dubnica nad Váhom, IL & 123 & 39,3 & 18 & $8 \times 8$ & $\hat{0}$ & 62 \\
\hline Filakovo,(P-272) City park & 191 & 60,9 & 18 & $14 \times 14$ & $\delta$ & 97 \\
\hline Filakovo, (P-272) City park & 173 & 55,2 & 20 & $16 \times 8$ & $\hat{\sigma}$ & 114 \\
\hline Fričovce (P-345), PO & 171 & 54,5 & 16 & $6 \times 5$ & $\hat{o}$ & 87 \\
\hline Galanta (P-146), GA & 285 & 90,5 & 20 & $18 \times 20$ & $\hat{0}$ & 144 \\
\hline
\end{tabular}


Second continuation of table 1

\begin{tabular}{|c|c|c|c|c|c|c|}
\hline Locality & $\begin{array}{c}\text { Trunk } \\
\text { circumference }(\mathrm{cm})\end{array}$ & $\begin{array}{c}\text { Trunk } \\
\text { diameter }(\mathrm{cm}) \\
\end{array}$ & $\begin{array}{c}\text { Tree height } \\
\text { (m) }\end{array}$ & $\begin{array}{c}\text { Crown } \\
\text { width }(\mathrm{m})\end{array}$ & $\begin{array}{c}\text { Gender } \\
(\circlearrowleft,+)\end{array}$ & Age \\
\hline Gbely, Art School & 80 & 25,5 & 8 & $4 \times 4$ & juv. & 41 \\
\hline Hájna Nová Ves (P-44), TO & 478 & 152,4 & 22 & $30 \times 30$ & q & 242 \\
\hline Hnúšt'a (SG), RS, $320 \mathrm{~cm}$ & 263 & 83,8 & 26 & $12 \times 15$ & $\hat{0}$ & 133 \\
\hline Hokovce - historical park & 166 & 52,8 & 16 & $14 \times 12$ & q & 84 \\
\hline Hokovce - historical park & 35 & 11 & 8 & $8 \times 4$ & juv. & 18 \\
\hline Hokovce - historical park & 36 & 12 & 8 & $8 \times 6$ & juv. & 18 \\
\hline Hokovce, private garden & 220 & 70,1 & 25 & $12 \times 14$ & $\sigma^{\pi}$ & 111 \\
\hline $\begin{array}{l}\text { Horenická Hôrka-Medné } \\
\text { (P-205), PU }\end{array}$ & 345 & 110,2 & 25 & $20 \times 20$ & 우 & 175 \\
\hline $\begin{array}{l}\text { Horné Obdokovce } \\
(P-50) \text {, TO }\end{array}$ & 192 & 61,2 & 25 & $12 \times 12$ & 0 & 97 \\
\hline $\begin{array}{l}\text { Horné Semerovce } \\
\text { (P-158), LV }\end{array}$ & 325 & 103,7 & 19 & $14 \times 10$ & 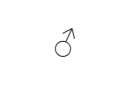 & 165 \\
\hline Hubice, historical park & 184 & 58,7 & 18 & $16 \times 12$ & $\widehat{\delta}$ & 93 \\
\hline Humenné (P-340), HE, & 275 & 87,6 & 22 & $15 \times 20$ & q & 139 \\
\hline Jaklovce (P-359), GL, $230 \mathrm{~cm}$ & 203 & 64,7 & 21 & $8 \times 8$ & q & 103 \\
\hline Janova Ves (P-42), T0 & 373 & 111,2 & 22 & $20 \times 20$ & $\widehat{0}$ & 197 \\
\hline $\begin{array}{l}\text { Jasov, monastery garden } \\
(\mathrm{P}-396), \mathrm{KE}\end{array}$ & 220 & 70,2 & 14 & $14 \times 14$ & $\hat{0}$ & 112 \\
\hline Kalinovo, park PS & 61 & 19,5 & 12 & $3 \times 3$ & juv. & 31 \\
\hline Kazimír (P-416),TV, 260 cm, double & $229 ; 170$ & $73 ; 54,2$ & 17 & $22 \times 20$ & q & 116 \\
\hline Klobušice (P-217), IL & 212 & 67,5 & 22 & $15 \times 15$ & 우 & 107 \\
\hline Klobušice (P-217), IL & 179 & 57,0 & 22 & $14 \times 14$ & $\widehat{0}$ & 91 \\
\hline Klobušice (P-217), historical park & 165 & 52,6 & 13 & $10 \times 5$ & ㅇ & 84 \\
\hline Kočovce (P-17), NM & 206 & 65,6 & 25 & $10 \times 10$ & q & 104 \\
\hline Komárno (P-187), KN & 76 & 24,2 & 12 & $10 \times 12$ & q & 38 \\
\hline Komjatice (P-166), NZ, $365 \mathrm{~cm}$ & 327 & 104,2 & 25 & $22 \times 22$ & q & 165 \\
\hline $\begin{array}{l}\text { Košice (P-382), } \\
\text { Botanical garden UPJŠ, KE }\end{array}$ & 69 & 22,0 & 12 & $5 \times 4$ & juv. & 35 \\
\hline $\begin{array}{l}\text { Košice (P-388), } \\
\text { Park J. A. Komenského, KE }\end{array}$ & 312 & 99,7 & 20 & $20 \times 20$ & $\hat{0}$ & 158 \\
\hline $\begin{array}{l}\text { Košice (P-390), FN, } \\
\text { KE Rastislavova St. (1) }\end{array}$ & 197 & 62,7 & 22 & $14 \times 16$ & q & 100 \\
\hline $\begin{array}{l}\text { Košice (P-390), FN, } \\
\text { KE Rastislavova St. (2) }\end{array}$ & 131 & 41,8 & 19 & $4 \times 0,5$ & q & 66 \\
\hline $\begin{array}{l}\text { Košice (P-390), FN, } \\
\text { KE, Rastislavova St., (4) }\end{array}$ & 121 & 38,5 & 12 & $11 \times 19$ & q & 61 \\
\hline $\begin{array}{l}\text { Košice (P-390), FN, } \\
\text { KE, Rastislavova St. (3) }\end{array}$ & 151 & 48,1 & 18 & $10 \times 12$ & $\hat{0}$ & 46 \\
\hline $\begin{array}{l}\text { Košice (P-390), FN, } \\
\text { KE, Rastislavova St. (5) }\end{array}$ & 133 & 42,5 & 11 & $10 \times 9$ & ㅇ & 68 \\
\hline Košice, Masarykova St. 3, ZŠ & 381 & 121,7 & 27 & $24 \times 12$ & 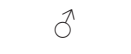 & 193 \\
\hline Košice, Town square MMM, KE & 15.5 & 5.0 & 5 & $2 \times 2$ & juv. & 8 \\
\hline Kovarce (P-48), TO & 167 & 53.2 & 19 & $12 \times 12$ & $\hat{\sigma}$ & 85 \\
\hline
\end{tabular}


Third continuation of table 1

\begin{tabular}{|c|c|c|c|c|c|c|}
\hline Locality & $\begin{array}{c}\text { Trunk } \\
\text { circumference }(\mathrm{cm})\end{array}$ & $\begin{array}{c}\text { Trunk } \\
\text { diameter }(\mathrm{cm})\end{array}$ & $\begin{array}{c}\text { Tree height } \\
\text { (m) }\end{array}$ & $\begin{array}{c}\text { Crown } \\
\text { width }(\mathrm{m})\end{array}$ & $\begin{array}{c}\text { Gender } \\
(\delta,+)\end{array}$ & Age \\
\hline $\begin{array}{l}\text { Krakovany, Park in the courtyard of the } \\
\text { company, PN }\end{array}$ & 40 & 12.8 & 7 & $3 \times 3$ & juv. & 20 \\
\hline $\begin{array}{l}\text { Kravany nad Dunajom } \\
\text { (P-189), KN }\end{array}$ & 189 & 60.5 & 16 & $13 \times 14$ & 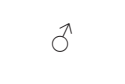 & 96 \\
\hline $\begin{array}{l}\text { Lučenec (SG), Ipel'ské tehelne Ltd., LC, } \\
\text { cv. 'Ohatsuki' }\end{array}$ & 267 & 85.1 & 22 & $16 \times 16$ & 우!! & 135 \\
\hline Lučenec, City park, LC & 40 & 12.0 & 12 & $8 \times 8$ & $\hat{0}$ & 19 \\
\hline Malacky (SG), MA & 16 & 5.3 & 5 & $3 \times 3$ & juv. & 10 \\
\hline Malacky (SG), MA & 18 & 6.0 & 7 & $3 \times 1$ & juv. & 10 \\
\hline Malacky, private garden (SG), MA & 59 & 18.8 & 12 & $7 \times 7$ & q & 20 \\
\hline Malinovo,(P-99) SC & 38 & 12.5 & 8 & $4 \times 4$ & $\hat{0}$ & 20 \\
\hline Malý Šariš (P-348), PO & $285 ; 315$ & $91 ; 100.7$ & 20 & $20 \times 20$ & $\hat{0}$ & 160 \\
\hline Martin (P-228), MT & 180 & 57.0 & 18 & $14 \times 10$ & $\hat{0}$ & 91 \\
\hline $\begin{array}{l}\text { Michalovce, park in front of the bank } \\
\text { VÚB, MI }\end{array}$ & 190 & 60.5 & 17 & $10 \times 16$ & $\hat{o}$ & 96 \\
\hline Modra (P-90), PK & 193 & 61.5 & 14 & $6 \times 6$ & 우 & 98 \\
\hline Mošovce (P-231), TR & 125 & 39.8 & 12 & $15 \times 14$ & $\hat{0}$ & 63 \\
\hline Motešice (P-13), TN & 110 & 35.0 & 15 & $12 \times 10$ & $\widehat{0}$ & 56 \\
\hline Necpaly (P-230), MT & 195 & 62.2 & 27 & $8 \times 8$ & 우 & 99 \\
\hline Nedožery-Brezany,168/120 & 48 & 15.3 & 10 & $5 \times 4$ & juv. & 24 \\
\hline Nenince (P-276), VK, $240 \mathrm{~cm}$ & 195 & 62.0 & 16 & $8 \times 8$ & $\hat{0}$ & 99 \\
\hline Nitra, Ďurčanského St. 12 & 60 & 19.2 & 5 & $6 \times 6$ & juv. & 31 \\
\hline Nitra, Faculty of Natur. Sci.UKF, NR & 25 & 8.0 & 6 & $3 \times 3$ & juv. & 13 \\
\hline Nitra, Kupecká ulica, alley & 6 pcs. & - & $4-5$ & $2 \times 2 ; 3 \times 3$ & juv. & 10 \\
\hline $\begin{array}{l}\text { Nitra, Nitra city park, } 1963, \mathrm{NR} \text {, triple } \\
\text { trunk }(234 \mathrm{~cm} / 74,5 \mathrm{~cm})\end{array}$ & $120 ; 119 ; 96$ & $38.2 ; 30.7$ & 18 & $18 \times 18$ & 우 & 60 \\
\hline Nitra, Nitra city park, 1963. NR & 81 & 26.0 & 12 & $14 \times 12$ & $\hat{\sigma}$ & 41 \\
\hline Nitra, Penzión LUx & 15 & 4.8 & 6 & $3 \times 3$ & juv. & 10 \\
\hline Nitra, rest. MALIBU & 107 & 34.2 & 15 & $9 \times 9$ & 우 & 54 \\
\hline Nitra, Špitálska St.1, NR & 102 & 32.5 & 12 & $9 \times 9$ & $\hat{0}$ & 52 \\
\hline Nová Dubnica, TN & $21 ; 33$ & $6.7 ; 10.8$ & $3.5 ; 4.5$ & $1 \times 1 ; 2 \times 2$ & juv. & 17 \\
\hline Nová Ves nad Žitavou (P-135), NR (1) & 228 & 72.7 & 16 & $18 \times 18$ & ㅇ & 116 \\
\hline Nová Ves nad Žitavou (P-135), NR (2) & 277 & 88.3 & 17 & $18 \times 18$ & 우 & 140 \\
\hline Nová Ves nad Žitavou (P-135), NR (4) & 172 & 54.8 & 20 & $2 \times 9$ & q & 87 \\
\hline Nová Ves nad Žitavou (P-135), NR & 202 & 64.4 & 22 & $16 \times 16$ & q & 102 \\
\hline Nový Život-Tonkovce, (P-191), DS & 97 & 31.0 & 15 & $3 \times 3$ & $\hat{0}$ & 49 \\
\hline Oponice (P-52), TO & 185 & 59.0 & 13 & $10 \times 8$ & $\hat{0}$ & 94 \\
\hline Palárikovo (P-174), NZ (4) & 247 & 78.6 & 20 & $14 \times 14$ & $\hat{0}$ & 125 \\
\hline Palárikovo (P-174), NZ (1) & 316 & 101.0 & 20 & $19 \times 19$ & $\hat{0}$ & 160 \\
\hline Palárikovo (P-174), NZ (2) & 62 & 19.7 & 10 & $6 \times 7$ & q & 31 \\
\hline Palárikovo (P-174), NZ (3) & 60 & 19.2 & 12 & $7 \times 5$ & 우 & 31 \\
\hline Palárikovo (P-174), NZ (5) & 100 & 31.9 & 10 & $0 \times 9$ & $\hat{0}$ & 51 \\
\hline Pieštany (SG), private garden, PN & 280 & 89.2 & 25 & $20 \times 20$ & $\hat{\jmath}$ & 142 \\
\hline
\end{tabular}


Fourth continuation of table 1

\begin{tabular}{|c|c|c|c|c|c|c|}
\hline Locality & $\begin{array}{c}\text { Trunk } \\
\text { circumference }(\mathrm{cm})\end{array}$ & $\begin{array}{c}\text { Trunk } \\
\text { diameter }(\mathbf{c m})\end{array}$ & $\begin{array}{l}\text { Tree height } \\
\text { (m) }\end{array}$ & $\begin{array}{c}\text { Crown } \\
\text { width }(\mathrm{m})\end{array}$ & $\begin{array}{c}\text { Gender } \\
(\lambda,+)\end{array}$ & Age \\
\hline $\begin{array}{l}\text { Piešt'any (P-58), Spa park near bath } \\
\text { "Eva", PN }\end{array}$ & 152 & 48.5 & 16 & $12 \times 10$ & 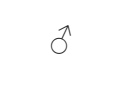 & 97 \\
\hline Piešt'any (P-58), Spa park, PN & 202 & 64.9 & 23 & $16 \times 14$ & q & 120 \\
\hline $\begin{array}{l}\text { Piešt'any (P-58), Spa park near outdoor } \\
\text { bath, PN }\end{array}$ & 186 & 59.3 & 25 & $16 \times 14$ & q & 120 \\
\hline Piešt'any, Park at High school, (118) PN & $76 ; 71 ; 27$ & $24.3 ; 22.7 ; 8$ & 15 & $6 \times 6$ & juv. & 37 \\
\hline Piešt’any, Park at high school,(116) PN & 82 & 26.2 & 15 & $8 \times 8$ & juv. & 41 \\
\hline Piešt’any, Park at high school,PN & 35 & $11.2 ; 12 ; 12.8$ & 10 & $4 \times 4$ & juv. & 17 \\
\hline Pohronský Ruskov (SG), LV, 300 cm & 261 & 83.2 & 14 & $23 \times 20$ & $\sigma^{\pi}$ & 132 \\
\hline Považ. Bystrica, at the cinema MIER & 48 & 15.5 & 4 & $4 \times 4$ & juv. & 50 \\
\hline Prešov, Art Garden, Po & 265 & 84.5 & 18 & $15 \times 15$ & $\hat{\sigma}$ & 134 \\
\hline Pribeník (P-418),TV & 330 & 105.5 & 25 & $16 \times 22$ & $\sigma^{\pi}$ & 168 \\
\hline Prievidza (SG), PD & 128 & 40.8 & 15 & $8 \times 8$ & 우 & 65 \\
\hline Rajecké Teplice (P-223), Spa park, ZA & 15 & 5.0 & 3 & $0,5 \times 0,5$ & juv. & 8 \\
\hline Rakovice (P-66), PN & 237 & 75.5 & 20 & $18 \times 15$ & ㅇ & 120 \\
\hline Rimavská Sobota (P-292), RS & 126 & 40.2 & 16 & $12 \times 12$ & q & 64 \\
\hline Rimavská Sobota, Športová St. č.4, RS & 135 & 43.0 & 10 & $8 \times 8$ & ㅇ & 68 \\
\hline Rimavská Sobota, Športová St. č.4, RS & 155 & 49.5 & 18 & $6 \times 10$ & $\sigma^{\pi}$ & 79 \\
\hline $\begin{array}{l}\text { Ružomberok, } \\
\text { Military hospital. }\end{array}$ & $120 ; 57$ & $38.2 ; 18.2$ & $14 ; 7$ & $8 \times 8$ & $\sigma^{\pi}$ & 95 \\
\hline Senica (SG), SE, alley-25 ks & $7-10-12$ & $4.8-9.8$ & $3-6$ & $2 \times 2$ & juv. & $8-16$ \\
\hline $\begin{array}{l}\text { Senica, Hviezdoslavova St., } 4 \text { pcs, } \\
\text { row of trees SE }\end{array}$ & $26-31$ & 8.4-9.8 & 7 & $2 \times 2$ & juv. & 16 \\
\hline Senné (SG), private garden, 1964, VK & 42 & 13.5 & 12 & $3 \times 4$ & juv. & 22 \\
\hline Sered', private garden & 59 & 18.8 & 12 & $7 \times 7$ & juv. & 30 \\
\hline $\begin{array}{l}\text { Slanec, Park around PS and KG, } \\
\mathrm{KE}, 362 \mathrm{~cm}\end{array}$ & 278 & 88.5 & 18 & $16 \times 14$ & $0^{\lambda}$ & 141 \\
\hline Slažany (P-118), ZM, & 118 & 37.7 & 16 & $10 \times 8$ & $\hat{\sigma}$ & 60 \\
\hline Slovenské Pravno, PS & 3 & 1.0 & 1 & $0,5 \times 0,5$ & juv. & 10 \\
\hline Spišská Belá, KK & 198 & 63.0 & 16 & $8 \times 9$ & $\hat{0}$ & 100 \\
\hline Stupava (P-96), MA & 195 & 62.2 & 20 & $12 \times 14$ & $\hat{\sigma}$ & 99 \\
\hline Súdovce (SG), KA & 302 & 96.5 & 14 & $12 \times 10$ & $\hat{0}$ & 153 \\
\hline Šamorín, private garden & 169 & 53.8 & 14 & $12 \times 12$ & $\hat{o}$ & 40 \\
\hline Šišov (P-34), BN & 160 & 51.0 & 28 & $8 \times 8$ & $\hat{\sigma}$ & 81 \\
\hline Šurianky (P-114), NR, $120 \mathrm{~cm}$ & 87 & 27.8 & 12 & $6 \times 0$ & $\hat{\sigma}$ & 44 \\
\hline Tomášikovo (P-148), GA, $430 \mathrm{~cm}$ & 315 & 100.4 & 25 & $16 \times 16$ & 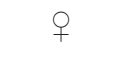 & 162 \\
\hline Tomášov (P-100), 362 cm & 290 & 90.7 & 20 & $20 \times 19$ & $\hat{\sigma}$ & 144 \\
\hline Topol'čany (P-40), TO & 110 & 35.0 & 17 & $6 \times 6$ & q & 56 \\
\hline Topol'čianky (P-115), ZM (1) & 225 & 71.7 & 20 & $16 \times 16$ & q & 114 \\
\hline Topol'čianky (P-115), ZM (2) & 305 & 97.5 & 12 & $14 \times 14$ & $\hat{\sigma}$ & 155 \\
\hline Topol'čianky (P-115), ZM (3) & 261 & 83.2 & 18 & $16 \times 16$ & q & 132 \\
\hline Topol'čianky (P-115), ZM (4) & 92 & 29.3 & 12 & $6 \times 6$ & q & 47 \\
\hline Tovarníky, (P-40), hist. park ObÚ & 157 & 50 & 18 & $3 \times 4$ & 우 & 79 \\
\hline
\end{tabular}


Fifth continuation of table 1

\begin{tabular}{|c|c|c|c|c|c|c|}
\hline Locality & $\begin{array}{c}\text { Trunk } \\
\text { circumference }(\mathrm{cm})\end{array}$ & $\begin{array}{c}\text { Trunk } \\
\text { diameter }(\mathrm{cm}) \\
\end{array}$ & $\begin{array}{l}\text { Tree height } \\
\text { (m) }\end{array}$ & $\begin{array}{c}\text { Crown } \\
\text { width }(\mathrm{m})\end{array}$ & $\begin{array}{c}\text { Gender } \\
(\circlearrowleft,+)\end{array}$ & Age \\
\hline Trávnica (P-169), NZ & 239 & 76.2 & 22 & $10 \times 8$ & q & 121 \\
\hline Trenčianske Teplice - Spa park $(35 \mathrm{~cm})$ & 19 & 6.2 & 4 & $0,5 \times 1$ & juv. & 10 \\
\hline Trenčianske Teplice - Spa park (54 cm) & 31 & 9.5 & 6 & $5 \times 5$ & juv. & 15 \\
\hline Trenčianske Teplice - Spa park $(95 \mathrm{~cm})$ & 64 & 20.4 & 12 & $8 \times 8$ & juv. & 32 \\
\hline Trenčín (P-7), TN & 290 & 92.7 & 16 & $10 \times 10$ & q & 147 \\
\hline Trenčín (P-7), TN & 365 & 116.7 & 16 & $12 \times 12$ & $0^{\lambda}$ & 185 \\
\hline Trenčín (P-7), TN & 210 & 67.0 & 17 & $10 \times 10$ & q & 106 \\
\hline Trnava (P-78), TT & 136 & 43.3 & 16 & $14 \times 12$ & $\sigma^{\pi}$ & 72 \\
\hline Trnava (P-78), TT & 116 & 36.9 & 14 & $6 \times 6$ & $0^{\pi}$ & 69 \\
\hline Trnava, (SG), Kalinčiakova St. 17, TT & 151 & 48.2 & 12 & $16 \times 14$ & q & 77 \\
\hline Turčianska Štiavnička (P-225), MT & 233 & 74.2 & 24 & $9 \times 9$ & q & 118 \\
\hline Vel'ký Blh (P-286), RS & 190 & 60.5 & 14 & $0 \times 14$ & q & 96 \\
\hline Vištuk, Park OÚ, PK & 22 & 7.0 & 5 & $5 \times 3$ & juv. & 11 \\
\hline Voderady (P-84) historical park, TT & 292 & 93 & 18 & $18 \times 18$ & $0^{\pi}$ & 148 \\
\hline Voderady (P-84), TT, $480 \mathrm{~cm}$ & 213 & 68.0 & 16 & $15 \times 15$ & 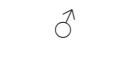 & 108 \\
\hline Vráble, private garden & 110 & 35.0 & 16 & $10 \times 10$ & 0 & 40 \\
\hline Záblatie (P-4),TN & 178 & 56.7 & 15 & $10 \times 12$ & q & 90 \\
\hline Zemianske Podhradie (P-12), NM & 219 & 70.0 & 24 & $16 \times 14$ & 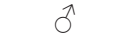 & 111 \\
\hline Zvolen, at the factory Bučina & 51 & 16.3 & 12 & $2 \times 2$ & juv. & 26 \\
\hline Zvolen, at the factory Bučina & 45 & 14.0 & 10 & $2 \times 2$ & juv. & 22 \\
\hline Zvolen, at the factory Bučina & 25 & 8.0 & 6 & $2 \times 1$ & juv. & 13 \\
\hline Želiezovce (P-162), LV & 195 & 62.0 & 16 & $15 \times 10$ & 0 & 98 \\
\hline $\begin{array}{l}\text { Žilina, at the Department Store MIRAGE, } \\
5 \text { pcs ZA }\end{array}$ & $25-29$ & $8.0-9.3$ & $4-5-6$ & $1 \times 1$ & juv. & 13 \\
\hline Župčany (P-347), PO & 136 & 43.3 & 16 & $9 \times 10$ & $\widehat{0}$ & 69 \\
\hline
\end{tabular}

Notes: P-44 - registration number of the historical park and garden, TO - Topolčany - district - according to the current territorial division, 19/1 - first half of the $19^{\text {th }}$ century, with the designation of the years -1850 of foundation of the dendrological building, SG - surrounding greenery, that is, the tree is in a territory other than a historical park or garden, PS - primary school, KG - kindergarten, juv. - an individual in juvenile growth

recorded 20 localities where no trees were found, but previously were identified by Benčat' (1992) or these are private and religious buildings, unavailable at the time of our research.

We included 45 ginkgo trees that reached their maximum dimensions. The group of trees with a trunk circumference of more than $400 \mathrm{~cm}$ included individuals in Hájná Nová Ves - $477 \mathrm{~cm}$; Bratislava Petržalka, Sady J. Krála $467 \mathrm{~cm}$. More numerous was the group of trees with trunk circumference over $300 \mathrm{~cm}$ (13 trees) and over $200 \mathrm{~cm}$ (33 trees).

\section{Some new findings on the cultivation and growth of Ginkgo biloba}

The number of localities with the intentional introduction of Ginkgo biloba in Slovakia cannot be complete, because in recent years, at the beginning of the $21^{\text {st }}$ century, other new plantings were added in various categories of urban greenery, but also in private gardens and parks.

During our research, we occasionally recorded the nesting of birds (garden turtle, gray crow, common magpie) on the trees of the two-lobed ginkgo. We found nesting cavities on tree trunks and skeletal branches, which cut down woodpeckers in soft wood (even healthy trees), or in the cracks of tree bark we found collected seeds of yew (Taxus baccata L.). We first discovered the natural rejuvenation and occurrence of ginkgo seedlings in Trenčín (2007), and later in Palárikovo (2011); Nitra - Nitra City Park (2011); Tomášikovo (2011), Bratislava - Botanical Garden (2011), Godrova St. no. 8 (2014); Košice - park around 
L. Pasteur Hospital (2014); Nová Ves nad Žitavou (2014). The best conditions for seed germination are in the fallen and accumulated leaves under the tree. The natural seed propagation (Figure 6) and occurrence of seedlings of foreign wood can be considered as the peak phase in the process of introduction, and in the case of ginkgo it was rare and rare.

When assessing the horticultural value and health of Ginkgo biloba trees, we did not find any major differences from typical habitual features (except for deformation and suppression of tree crowns, in a group or under the crowns of other surrounding trees), so we rated the trees with the highest number of points ( $5^{\text {th }}$ grade). We did not record the occurrence of animal pests (especially leaf-eating, sucking, and wooddestroying insect pests) or fungal diseases on ginkgo trees during this research, and we also evaluated them at the highest level 5. The only disadvantage that will probably limit the cultivation of trees in our conditions is the unpleasant smell (after rotting meat) of ripening and falling fruit from female individuals. There have already been cases of tree felling for hygienic and safety reasons if the fruit pollutes busy streets, but especially pedestrian sidewalks, and there is a risk of slipping and possible fall of pedestrians. As a precautionary measure, we recommend covering the sidewalks under the fruiting trees, making structures covered with foil or tarpaulin with a tendency to the fruiting trees, permanent removal of deciduous fruit from the

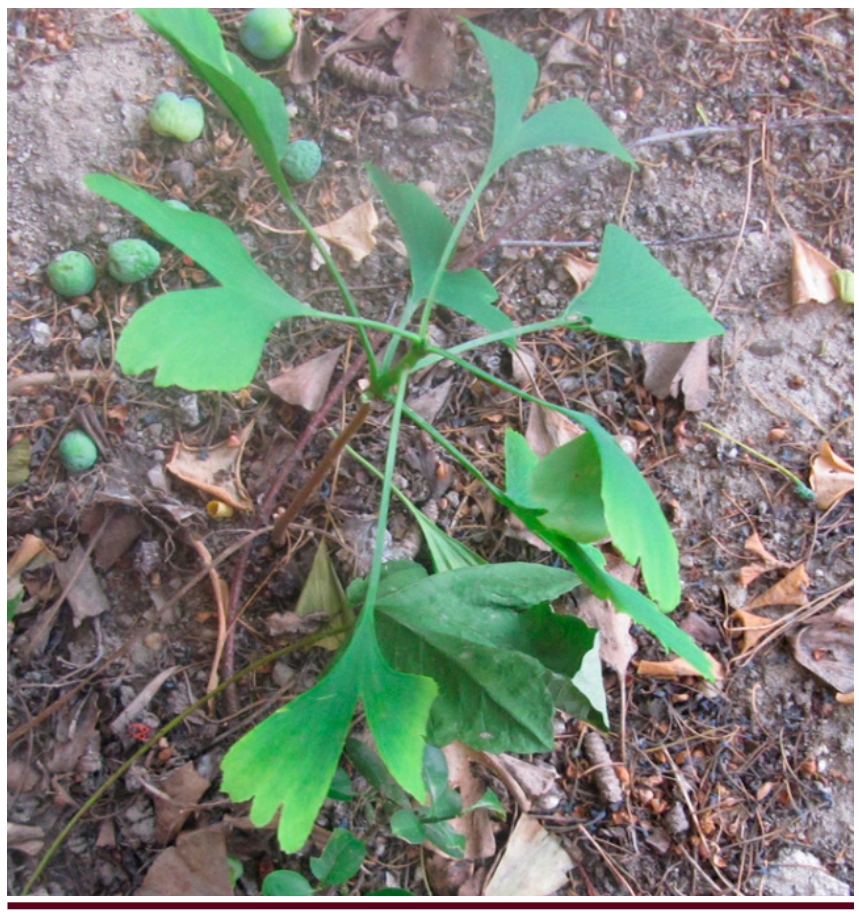

Figure 6 Natural seed propagation under the ginkgo tree Photo by Pavel Hrubík

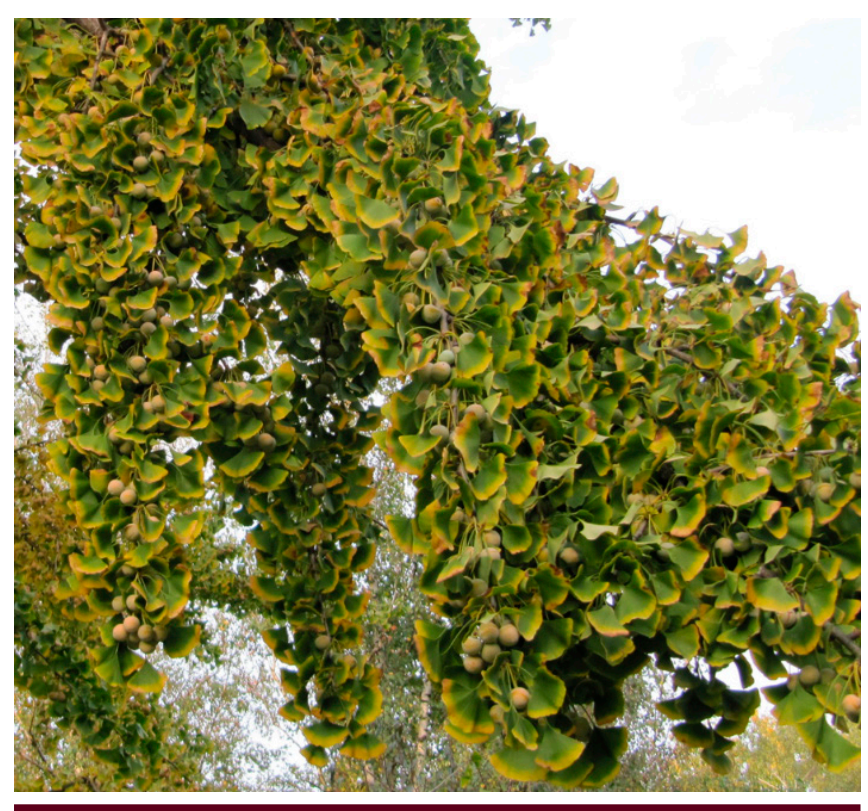

Figure 7 Large fruits observed on female ginkgo tree grown in the city park in Nitra Photo by Pavel Hrubík

sidewalks, especially in parks, front gardens, and street plantings.

During our field research, we also recorded certain morphological differences (habitual features on trees -

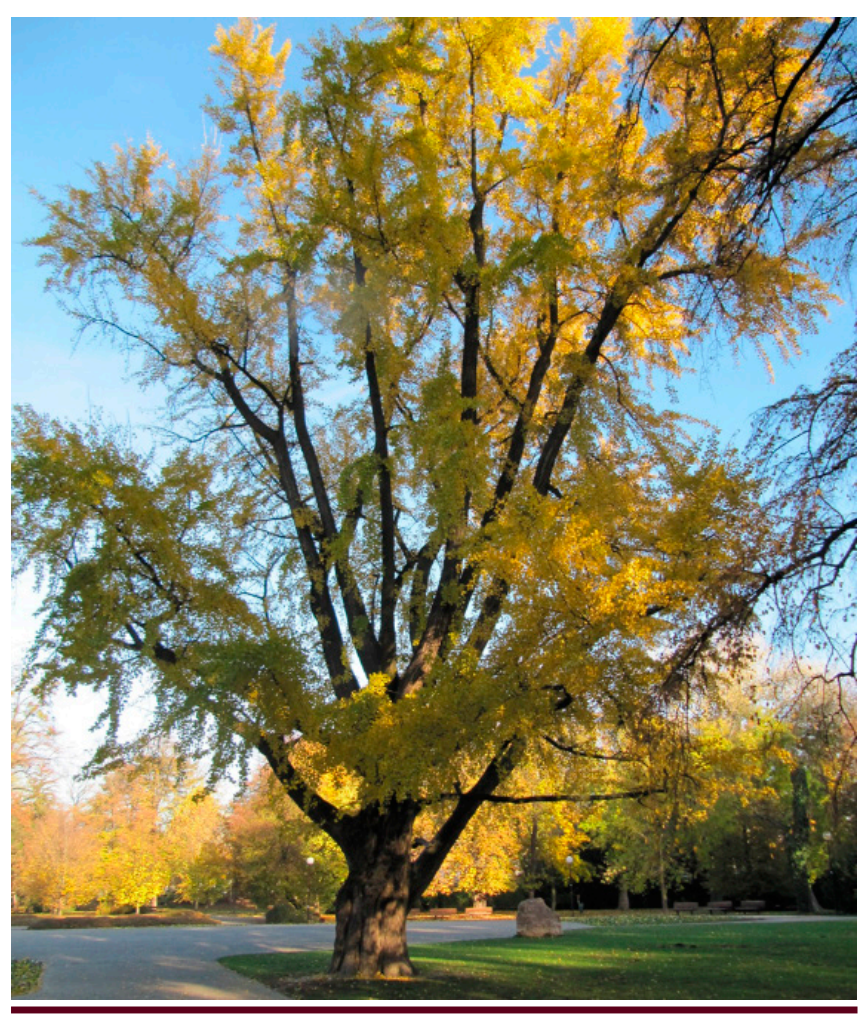

Figure 8 Distinctive golden-yellow colour of leaves in autumn in the locality Bratislava - Gardens of J. Král'

Photo by Pavel Hrubík 


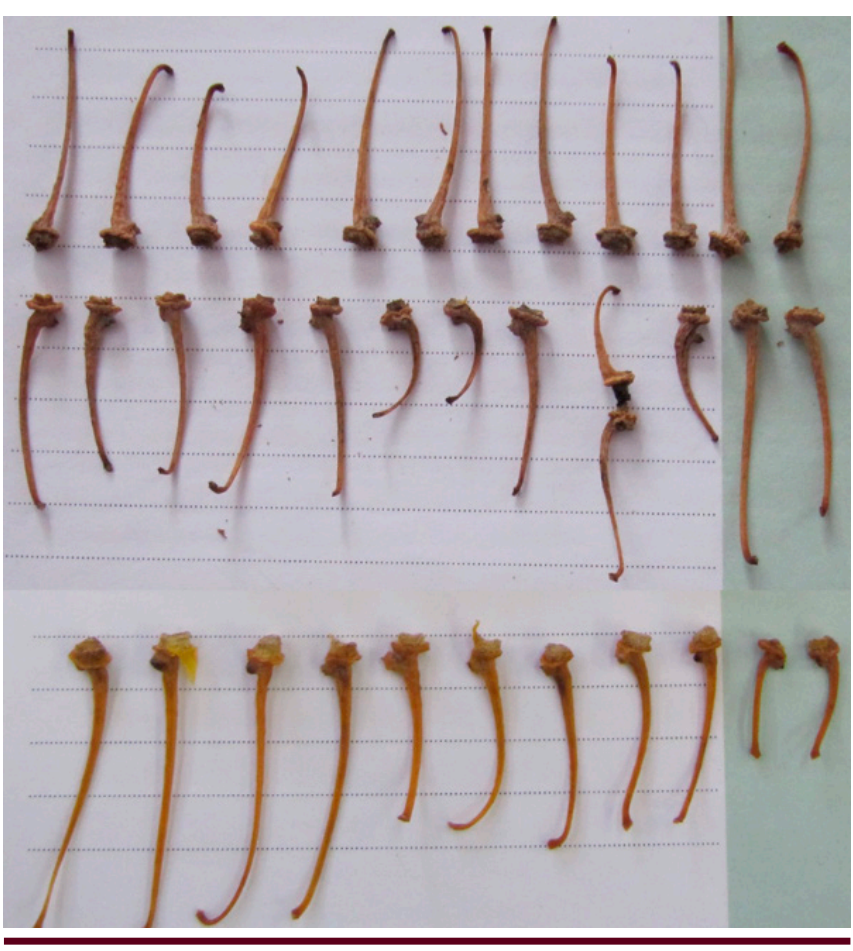

Figure 9 The variability of fruits stalks Photo by Pavel Hrubík

branching and shape of the crown; angle of protrusion and growth of lateral branches from the main trunk; leaf fall time; tree sex differences). Large fruits on long stems, we recorded on trees in Kočovce, Rimavská Sobota, Nová Ves nad Žitavou, Topol'čianky, Nitra, Bratislava - Botanical Garden (Figure 7). Distinctive golden-yellow colour of leaves in autumn in localities Kočovce, Nová Ves nad Žitavou, Topolčianky, Trenčín, Bratislava - Gardens of J. Král' in Petržalka, Dunajská

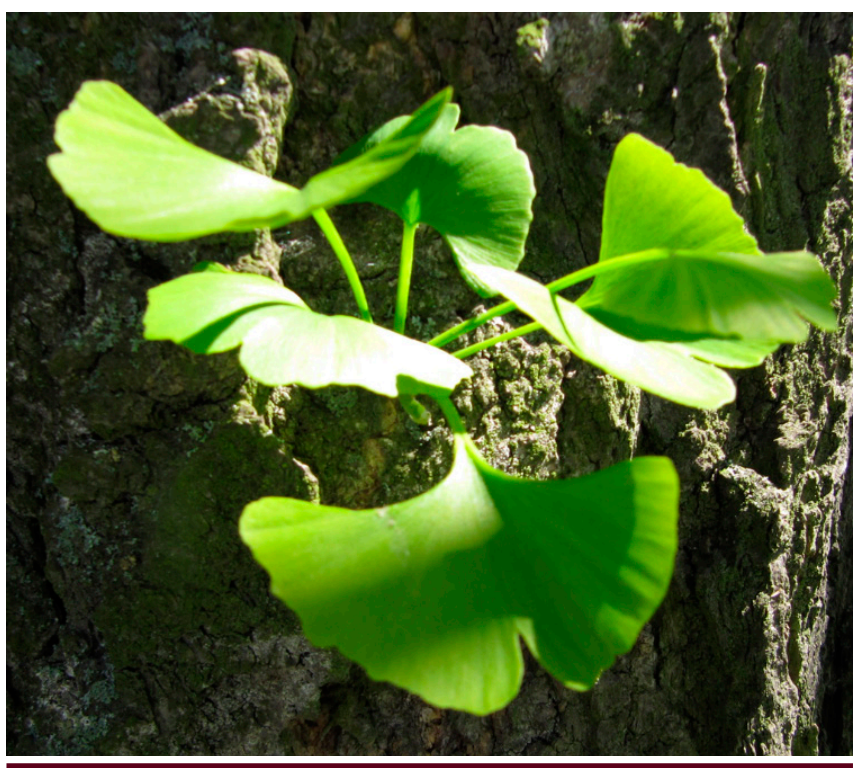

Figure 10 Epicormic sprout on the ginkgo tree trunk Photo by Pavel Hrubík

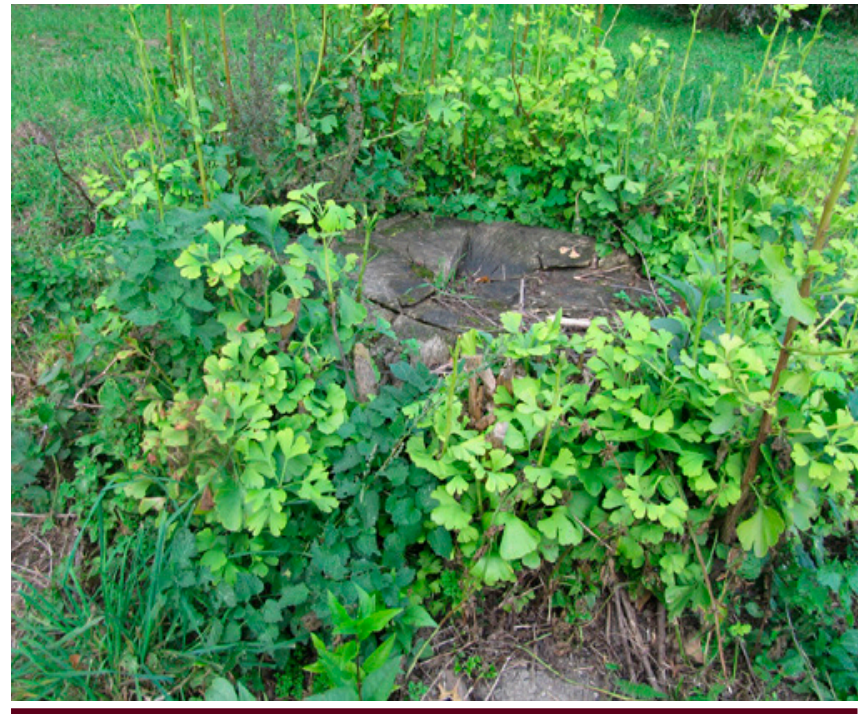

Figure 11 Root stalks growing on the trunk of a felled ginkgo tree in Trenčín Photo by Pavel Hrubík

street (Figure 8). A special peculiarity was the autumn in 2014, when during our research on September, October, and mid-November, the ginkgo trees kept the green leaves in the entire crown of the tree. At the same time, there are also differences between localities

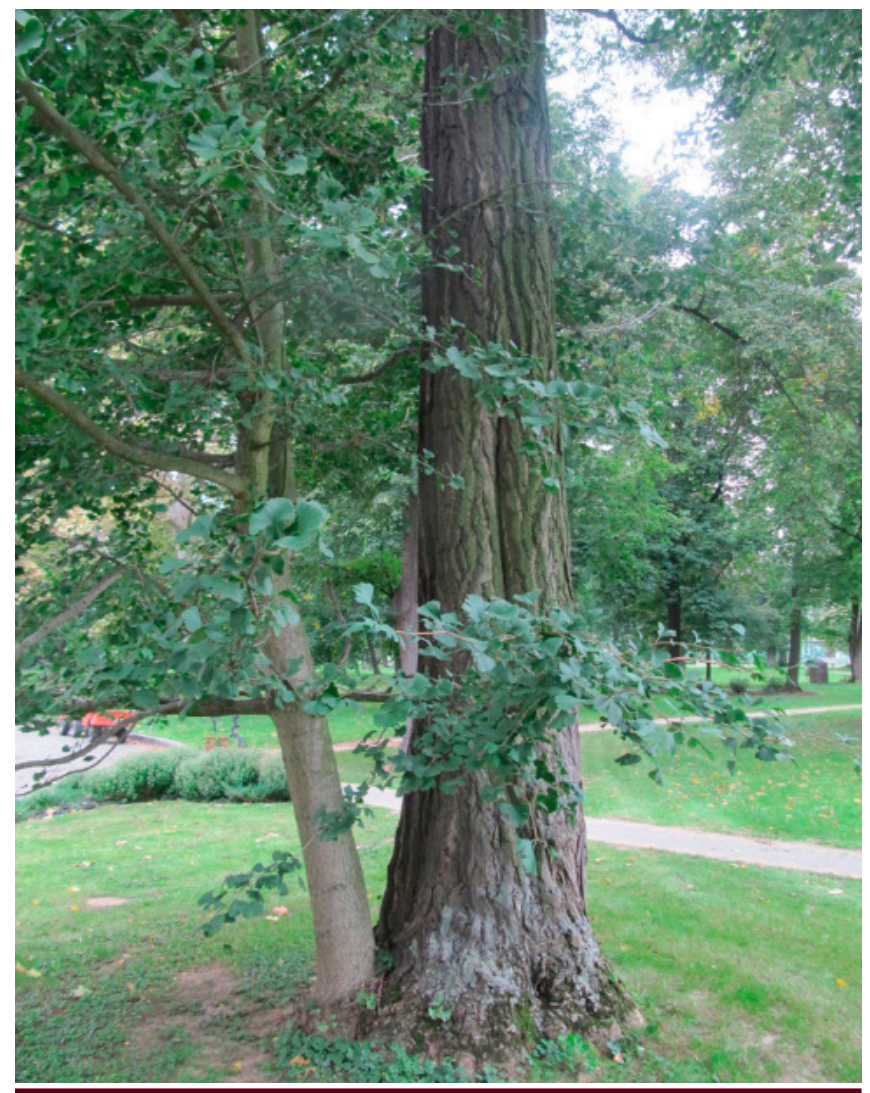

Figure 12 A lateral branch formed after mechanical damage to the ginkgo strain in the locality Trávnica Photo by Pavel Hrubík 


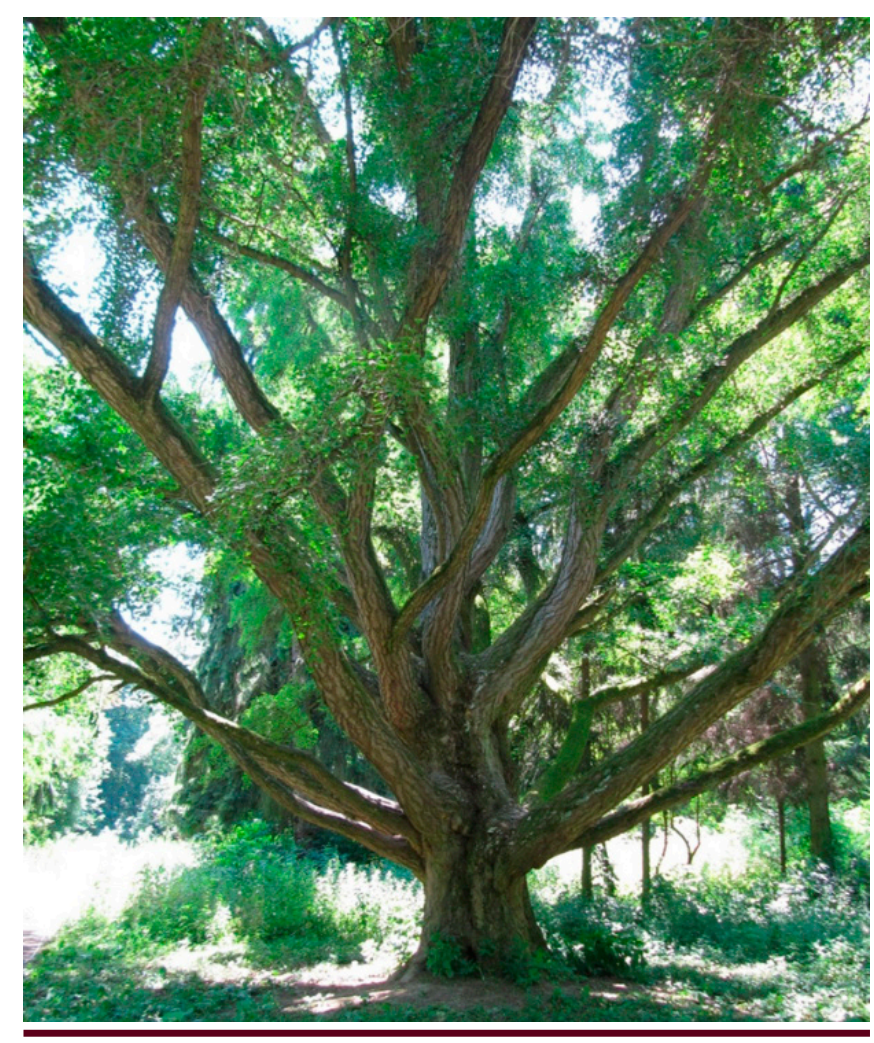

Figure 13 Heavily branched ginkgo tree with trunk circumference $478 \mathrm{~cm}$ in the locality Hajná Nová Ves. The oldest tree of Ginkgo biloba in Slovakia Photo by Pavel Hrubík

in Slovakia from one year to another. The prevailing finding was that the leaves on the male trees fall off earlier than on the female fruiting trees (preservation of the leaves during fruit ripening is also more logical). In 2014, this did not manifest itself in Nitra - Nitra City Park, because in mid-November, the leaves on the female tree were fallen, while on the neighboring male tree, all the leaves remained golden-yellow on the tree.

The size of the leaves is very variable, sometimes the length of the leaf stalk is extreme $(6.2-7.6 \mathrm{~cm}$ in Kočovce) (Figure 9), the leaves are deeply lobed, large, fleshy, they are on the stem and roots samplings of the trees (Figure 10), $(15.5 \times 13.5 \mathrm{~cm}$, leaf lobe depth up to $8 \mathrm{~cm}$, leaf size $12 \times 14.8,11 \times 13.5 \mathrm{~cm}$, length of samplings $100-150-180 \mathrm{~cm}$, more than 220 pieces of root stalks around the trunk circumference of a felled tree in Trenčín).

The occurrence of samplings on the stems and roots of Ginkgo biloba trees is extremely strong. This was most pronounced in Trenčín, after the felling of a male tree on the construction site of the future hotel in 2008. The following spring, we found massive 100-150-180 long shoots on the trunk of the tree, more than 220 pieces around the entire circumference of the trunk. The leaves
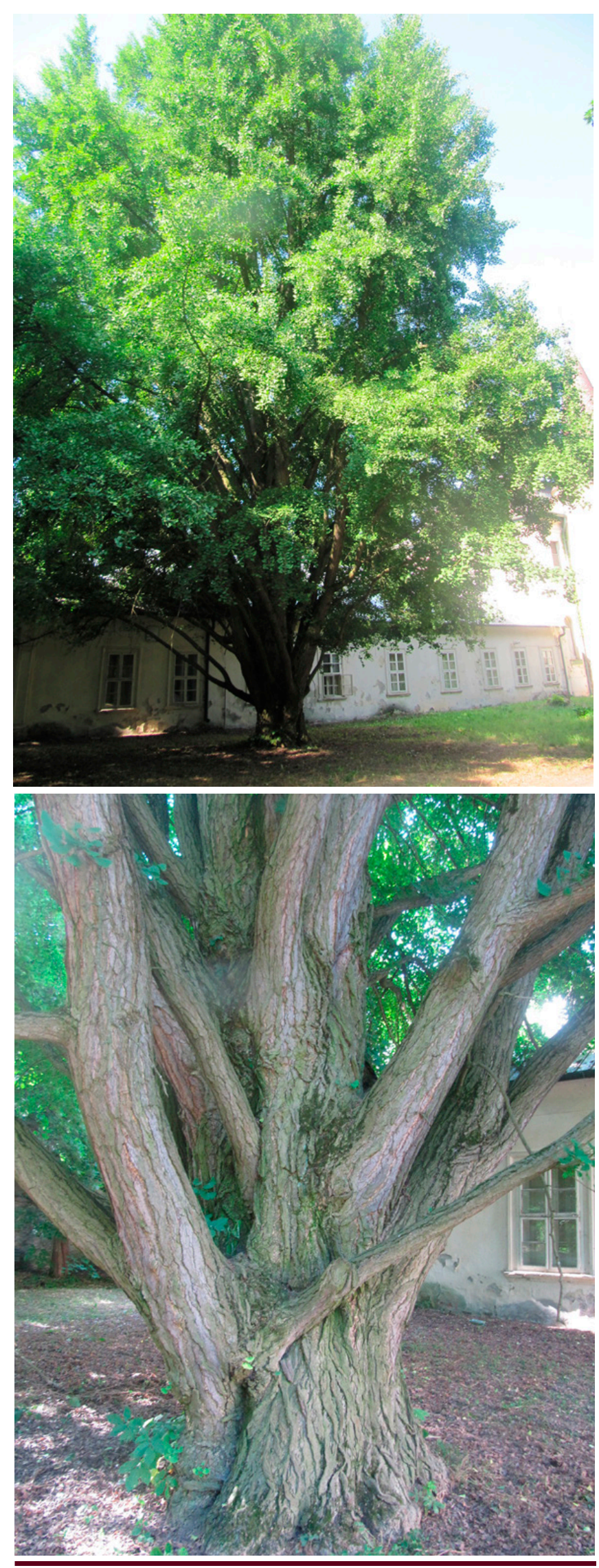

Figure 14 Ginkgo tree in Janova Ves with unique branching and trunk circumference $373 \mathrm{~cm}$ Photo by Pavel Hrubík 


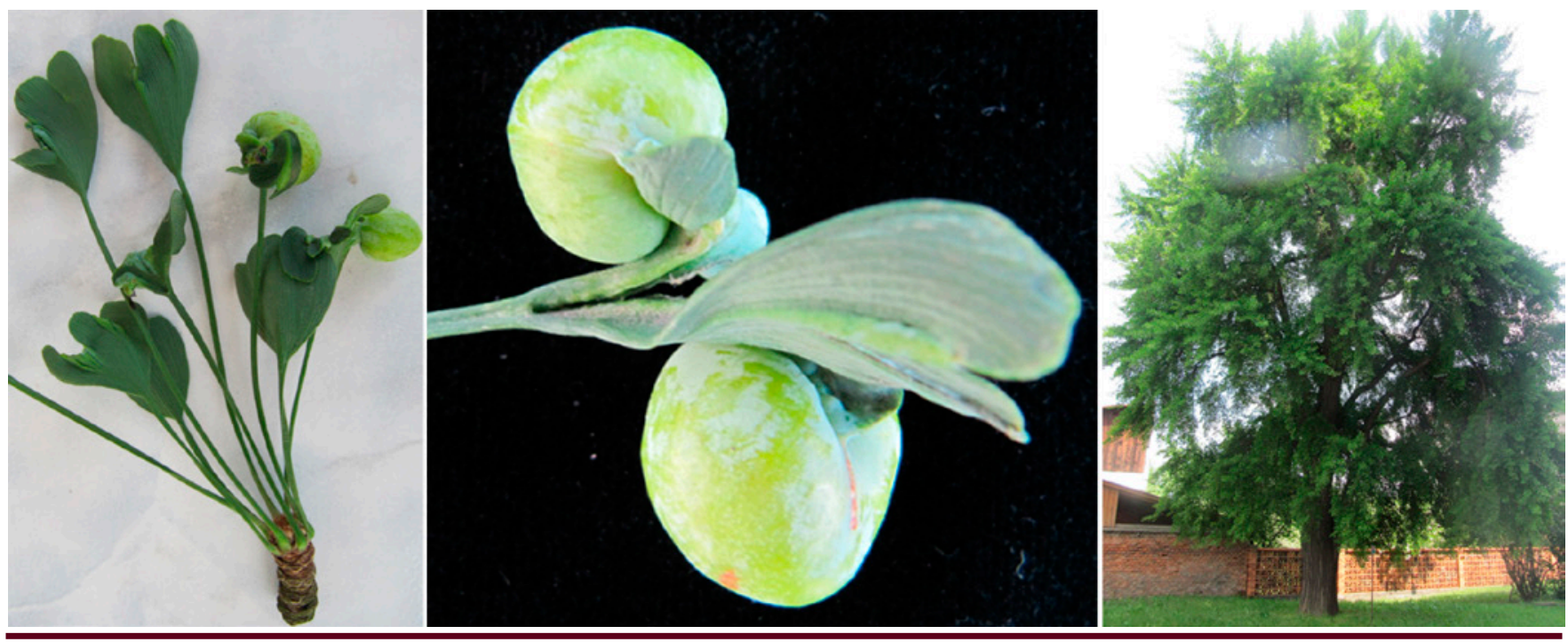

Figure 15 Observation of the occurrence of fruits on the leaves, referred to as cv. Ohatsuki of ginkgo growing in Lučenec Photos by Pavel Hrubík and Katarína Ražná

on the samplings were large, fleshy, deeply lobed; bark on young shoots cinnamon brown, with pronounced brown lenticules, shoots 1-2 years old (Figure 11). Stump youth also occurred on felled trees in Košice; on the sawed branches and on the trunk of the Ginkgo biloba in Horné Semerovce (the budding shoots were also on the cut pieces of the trunk lying on the ground under the tree). It also regenerates branches broken by the wind, snow, or the weight of ripening fruits (Topolčianky). It does not tolerate shading, limiting the growth of the crown by climbing trees (Hedera helix L. - Arborétum Mlyňany, Pieštany, Senné).

Also important is the rapid healing of wounds after mechanical damage to the trunk and branches, sawing of branches in the crown of the tree, sawing of the dry terminal top of the tree (then a beautiful, fan-shaped crown and top of the side branches - Trávnica); very good and fast is "hardening" after trimming thinner branches and healing larger wounds on the tree trunk (Figure 12).

In terms of the vertical distribution of Ginkgo biloba in Slovakia, we consider the highest-lying locality Ginkgo biloba, trees growing in the Botanical Garden of the Secondary Vocational Forestry School in Banská Štiavnica (three trees, one of them fruiting, female tree); the lowest localities included the ginkgo trees in Kravany pri Dunaji, Komárno, Pribeník, and Kazimír.

It is worth mentioning some of the particularly special trees, specific to twin habits, trunk dimensions, and fruits on leaves (Figures 13-15).

In the end, we present a list of the oldest trees of Ginkgo biloba in Slovakia (Table 3). The data on trees dimensions and age come from the period of our research in 2016.

On the importance of recognizing genetic diversity of Ginkgo biloba populations pointed out several studies focused on applying different molecular markers as genomic microsatellites (Yan et al., 2006; Yan et al., 2009; Li et al., 2009; Xie et al., 2013), RAPD (Fan et al., 2004; Li et al., 2013; Mei et al., 2014), ISSR (Mei et al., 2014), RFLP (Shen et al., 2005) and microsatellites (Xu et al., 2015) markers based on chloroplast DNA and microRNA-based markers (Ražná et al., 2020). Attention is also paid to the gender differentiation of ginkgo trees at the molecular level (Jiang et al., 2003; Liao et al., 2009; Milewicz and Sawicki, 2013).

In order to establish a molecular base to understand the evolution of ginkgo and to resolve the ambiguous phylogenetic relationship of ginkgo among the gymnosperm, several studies have been performed (Brenner et al., 2005; Lin et al., 2011; Lin et al., 2012; Šmarda et al., 2016).

Whereas ginkgo leaves contain a quantity of medicinally valuable compounds, extensive studies are carried on the identification and characterization of genes and molecules connected to these biosynthetic processes (Tekel'ová et al., 2006; Zittlau, 2007; Wang et al., 2010; Han et al., 2015; He et al., 2015; Wang et al., 2015).

The genetic background of ginkgo resistance to a wide spectrum of biotic and abiotic stress conditions was also analysed (Mohanta, 2012). In vitro approaches of ginkgo micropropagation are known from the literature (Tommasi and Scaramuzzi, 2004; Mantovani et al., 2013). 
Table 3 The oldest Ginkgo biloba L. trees in Slovakia (2016)

\begin{tabular}{|c|c|c|c|c|c|c|}
\hline Locality & $\begin{array}{c}\text { Trunk } \\
\text { circumference }(\mathrm{cm})\end{array}$ & $\begin{array}{c}\text { Trunk } \\
\text { diameter }(\mathrm{cm})\end{array}$ & $\begin{array}{l}\text { The height of } \\
\text { the tree }(\mathrm{m})\end{array}$ & $\begin{array}{c}\text { Crown } \\
\text { width }(\mathrm{m})\end{array}$ & $\begin{array}{l}\text { Gender } \\
(\mathrm{M}, \mathrm{F})\end{array}$ & Age \\
\hline $\begin{array}{l}\text { Hajná Nová Ves (P-44), T0, } \\
\text { 19/1-1850 }\end{array}$ & 478 & 152.4 & 22 & $30 \times 30$ & $\mathrm{~F}$ & 242 \\
\hline $\begin{array}{l}\text { Bratislava, J. Král' Gardens, BA, } \\
\text { (P-110), 18/2 -1751 }\end{array}$ & 467 & 148.8 & 20 & $28 \times 24$ & $\mathrm{~F}$ & 236 \\
\hline Janova Ves (P-42), T0,19/2-1851 & 373 & 111.2 & 22 & $20 \times 20$ & M & 197 \\
\hline $\begin{array}{l}\text { Košice, Masarykova St.3, PS, KE, } \\
\text { 19/1-1850 }\end{array}$ & 381 & 121.7 & 27 & $24 \times 12$ & M & 193 \\
\hline $\begin{array}{l}\text { Častá (Červený Kameň) } \\
\text { (P-424, PK,18/2-1771 }\end{array}$ & 375 & 122.2 & 14 & $12 \times 14$ & $\mathrm{~F}$ & 194 \\
\hline Pribeník (P-418), TV, 19/1-1850 & 330 & 105.5 & 25 & $16 \times 22$ & M & 168 \\
\hline $\begin{array}{l}\text { Komjatice (P-166), NZ, 18/2-1751 } \\
(365 \mathrm{~cm})\end{array}$ & 327 & 104.2 & 25 & $22 \times 22$ & $\mathrm{~F}$ & 165 \\
\hline $\begin{array}{l}\text { Horné Semerovce (P-158), LV, } \\
\text { 20/1-1950 }\end{array}$ & 325 & 103.7 & 19 & $14 \times 10$ & M & 165 \\
\hline $\begin{array}{l}\text { Tomášikovo (P-148), GA, } \\
\text { 8/2-1751, (430 cm) }\end{array}$ & 315 & 100.4 & 25 & $16 \times 16$ & $\mathrm{~F}$ & 162 \\
\hline $\begin{array}{l}\text { Palárikovo (P-174), NZ, } \\
\text { 19/1- } 1850\end{array}$ & 316 & 101.0 & 20 & $19 \times 19$ & M & 160 \\
\hline $\begin{array}{l}\text { Malý Šariš (P-348, PO, } \\
\text { 19/1 - } 1850\end{array}$ & $315 ; 285$ & $100.7 ; 91.0$ & 20 & $20 \times 20$ & M & 160 \\
\hline $\begin{array}{l}\text { Horenická Hôrka-Medné } \\
\text { (P-205, PU, 19/2-1851 }\end{array}$ & 345 & 110.2 & 25 & $20 \times 20$ & $\mathrm{~F}$ & 175 \\
\hline $\begin{array}{l}\text { Košice, Park J. A. Komenský } \\
\text { (P-388),KE }\end{array}$ & 312 & 99.7 & 20 & $20 \times 20$ & M & 158 \\
\hline $\begin{array}{l}\text { Voderady (P-84), TT, 19/2-1851 } \\
(480 \mathrm{~cm})\end{array}$ & 292 & 93.0 & 18 & $18 \times 18$ & M & 148 \\
\hline Súdovce, KA, 20/1 - 1950 & 302 & 96.5 & 14 & $12 \times 10$ & M & 153 \\
\hline $\begin{array}{l}\text { Topol'čianky (P-115). ZM, } \\
\text { 18/2 - } 1751\end{array}$ & 305 & 97.5 & 12 & $14 \times 14$ & M & 155 \\
\hline $\begin{array}{l}\text { Bojnice (P-1; 2; 3), PD, 19/1-1850, } \\
(415 \mathrm{~cm})\end{array}$ & 290 & 92.4 & 26 & $12 \times 12$ & M & 147 \\
\hline $\begin{array}{l}\text { Tomášov (P-100), SC, 18/2-1751 } \\
(362 \mathrm{~cm})\end{array}$ & 290 & 90.7 & 20 & $20 \times 19$ & M & 144 \\
\hline Galanta (P-146), GA, 19/2-1851 & 285 & 90.5 & 20 & $18 \times 20$ & M & 144 \\
\hline Piešt'any, PN, 19/1-1850 (SG) & 280 & 89.2 & 25 & $20 \times 20$ & M & 142 \\
\hline $\begin{array}{l}\text { Slanec, PS, KG, KE, 19/1-1850 } \\
(362 \mathrm{~cm})\end{array}$ & 278 & 88.5 & 18 & $16 \times 14$ & M & 141 \\
\hline $\begin{array}{l}\text { Nová Ves nad Žitavou (P-135), NR, } \\
19 / 1-1850\end{array}$ & 277 & 88.3 & 18 & $22 \times 24$ & $\mathrm{~F}$ & 140 \\
\hline Bojnice, PD, 19/1-1850 & 276 & 87.9 & 21 & $10 \times 10$ & M & 140 \\
\hline Humenné (P-340), HN, 18/2 & 275 & 87.6 & 22 & $15 \times 20$ & $\mathrm{~F}$ & 139 \\
\hline 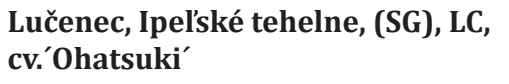 & 267 & 85.1 & 22 & $16 \times 16$ & $\mathrm{~F}$ & 135 \\
\hline $\begin{array}{l}\text { Topol'čianky (P-115), ZM, 18/2- } \\
1751(293 \mathrm{~cm})\end{array}$ & 261 & 83.2 & 18 & $16 \times 16$ & $\mathrm{~F}$ & 132 \\
\hline $\begin{array}{l}\text { Pohronský Ruskov (SG), LV, 20/1- } \\
1950(300 \mathrm{~cm})\end{array}$ & 261 & 83.2 & 14 & $23 \times 20$ & M & 132 \\
\hline
\end{tabular}


Continuation of table 3

\begin{tabular}{|c|c|c|c|c|c|c|}
\hline Locality & $\begin{array}{c}\text { Trunk } \\
\text { circumference }(\mathrm{cm})\end{array}$ & $\begin{array}{c}\text { Trunk } \\
\text { diameter }(\mathrm{cm})\end{array}$ & $\begin{array}{l}\text { The height of } \\
\text { the tree }(\mathrm{m})\end{array}$ & $\begin{array}{c}\text { Crown } \\
\text { width }(\mathrm{m})\end{array}$ & $\begin{array}{c}\text { Gender } \\
(\mathrm{M}, \mathrm{F})\end{array}$ & Age \\
\hline Beladice (P-124), ZM, 19/2-1851 & 259 & 82.5 & 18 & $16 \times 16$ & M & 131 \\
\hline $\begin{array}{l}\text { Hnúšt'a (SG), RS, 20/1-1950 } \\
(320 \mathrm{~cm})\end{array}$ & 263 & 83.8 & 26 & $12 \times 15$ & M & 133 \\
\hline $\begin{array}{l}\text { Palárikovo (P-174), NZ, } \\
19 / 1 \text { - } 1850\end{array}$ & 247 & 78.6 & 20 & $14 \times 14$ & M & 125 \\
\hline Trávnica (P-174), NZ, 18/2- 1751 & 239 & 76.2 & 22 & $10 \times 8$ & $\mathrm{~F}$ & 121 \\
\hline Rakovice (P-66), PN, 19/2 - 1851 & 237 & 75.5 & 20 & $18 \times 15$ & $\mathrm{~F}$ & 120 \\
\hline Beladice (P-124), ZM, r. - 130 r. & 237 & 75.2 & 20 & $16 \times 16$ & $\mathrm{~F}$ & 130 \\
\hline Abramová (SG), TR, 18/2-1751 & 238 & 76.0 & 14 & $18 \times 14$ & $\mathrm{~F}$ & 121 \\
\hline Betliar (P-407), RV, 19/1 - 1850 & 233 & 74.3 & 19 & $19 \times 14$ & M & 118 \\
\hline Trenčín (P-7), TN, 19/1 - 1850 & 365 & 116.7 & 16 & $12 \times 12$ & M & 185 \\
\hline $\begin{array}{l}\text { Turčianska Štiavnička (P-225). } \\
\text { MT, 18/2-1751 }\end{array}$ & 233 & 74.2 & 24 & $9 \times 9$ & $\mathrm{~F}$ & 118 \\
\hline $\begin{array}{l}\text { Kazimír (P-416), TV, 19/1-1850 } \\
(260 \mathrm{~cm})\end{array}$ & $229 ; 170$ & $73.0 ; 54.2$ & 17 & $22 \times 20$ & $\mathrm{~F}$ & 116 \\
\hline $\begin{array}{l}\text { Nová Ves nad Žitavou (P-135), NR, } \\
\text { 19/1-1850 }\end{array}$ & 228 & 72.7 & 16 & $23 \times 20$ & $\mathrm{~F}$ & 115 \\
\hline $\begin{array}{l}\text { Topol'čianky (P-115), ZM, } \\
\text { 18/2-1751 }\end{array}$ & 225 & 71.7 & 20 & $16 \times 16$ & $\mathrm{~F}$ & 113 \\
\hline Trenčín (P-7), TN, 19/1-1850 & 290 & 92.7 & 16 & $10 \times 10$ & $\mathrm{~F}$ & 147 \\
\hline Prešov, Garden of Art (SG) & 265 & 84.5 & 18 & $15 \times 15$ & M & 134 \\
\hline Jasov, Monastery Garden & 220 & 70.2 & 14 & $14 \times 14$ & M & 112 \\
\hline Hokovce, private garden & 220 & 70.1 & 25 & $12 \times 14$ & M & 180 \\
\hline Bratislava, Bot. garden & 222 & 70.7 & 16 & $8 \times 8$ & $\mathrm{~F}$ & 112 \\
\hline
\end{tabular}

Notes: P-44 - registration number of the historical park and garden, TO - Topolčany - district - according to the current territorial division, 19/1 - first half of the $19^{\text {th }}$ century, with the designation of the years -1850 of foundation of the dendrological building, SG - surrounding greenery, that is, the tree is in a territory other than a historical park or garden, PS - primary school, KG - kindergarten

During our long-term research of Ginkgo biloba and its cultural distribution in Slovakia, we verified or confirmed the knowledge about the growth, cultivation, use of fruits and seeds of this rare foreign tree.

Ginkgo biloba is a dioecious tree. Determining and differentiating the sex of trees is difficult, until the time of flowering and fruiting trees, is practically impossible. Several published findings concerning the gender differentiation, morphological and physiological features are known (Benčast', 1982; Pagan and Randuška, 1988; Tomaško, 2004; Benčat', 2009; van Beek, 2000; Begovic, 2011; Kwant, 2011; Zhang et al., 2015). However, based on our long-term research and experiences obtained from three dendrological expeditions in the Democratic People's Republic of Korea (1983 and 1985) and China (1998), we have also come to conflicting views and experiences:

- The male and female trees occur in a ratio $1: 1$. Based on our research and practical experience, we cannot confirm this, and we observed that males predominate in the population.

- Habitus of the tree: male tree - side branches protruding at an acute angle from the main trunk; female tree - horizontally - horizontally projecting lateral branches from the trunk of the tree (almost at right angles). To our knowledge, it is exactly the opposite. The male tree has branches protruding horizontally (almost at right angles). The female tree has branches protruding from the main trunk at an acute angle.

- Number of grooves (ribs) per seed: seeds from which male trees grow have 3 ribs; those producing female tree - 2 ribs. To our knowledge, this phenomenon has the opposite character. The seeds from which male trees grow have 2 ribs on the seed (these are in absolute predominance); the ones producing female tree has 3 ribs per seed (a rare occurrence). 
- The male trees bloom 2-3 weeks earlier than female ones. Due to the distances between the research localities of ginkgo trees, we did not perform regular phenological monitoring, so we cannot confirm the previous thesis, but we can agree with the data.

- Gender differentiation of Ginkgo biloba trees can be determined by the depth of cuts on the leaf blade: male trees have a deep cut on the leaf blade; female trees have a shallow notch at the leaves, up to entire leaves. We cannot confirm this thesis with certainty, moreover, the variability of the leaves on the one tree is very great.

- Fallen leaves in autumn: male trees tend to fall earlier; female trees have a later date of leaf fall. We can agree with this thesis, but in 2014, in Nitra City Park, the leaves of the male tree were kept two weeks longer (and fell later, while the female tree had been without leaves for a long time).

\section{Conclusions}

The final analysis of the research results on the cultigenous area of Ginkgo biloba in Slovakia confirmed the occurrence of this rare tree in more than one hundred localities (103 localities). No trees were found in the 18 previously registered localities of the occurrence of ginkgo, 5 trees were felled for various reasons (hygienic, safety, other reasons). The number of trees in solitary plantings (or in groups of three trees) reached 203 trees. 89 trees were planted in tree lines and alley plantings (especially in the city streets). There were 73 male trees, 65 female and 154 pregenerative individuals, a total of 292 trees of Ginkgo biloba. The number of ginkgo trees decreases in the following row: Bratislava - 42 trees; Senica - 27; Zlaté Moravce - 26; Nitra - 10; Košice - 9; Pieštany - 9; Topoľčany - 8; Žilina. 7; Nové Zámky - 7; in other districts (32) only 1-3 trees grow; we did not detect Ginkgo biloba trees in 31 districts. We evaluated the basic dendrometric and growth parameters of the oldest ginkgo trees, found in Slovakia. We evaluated 42 trees (aged 242 - 111 years) in 35 localities.

\section{Conflict of interests}

Authors declare no conflict of interests.

\section{Ethical statement}

This article does not contain any studies that would require an ethical statement.

\section{Acknowledgements}

We would like to thank our families for their support and patience. We thank the assoc. prof. L'uba Ďurišová, $\mathrm{Ph} . \mathrm{D}$. for consulting the terminology.

\section{References}

Begovic, B.M. (2011). Nature's Miracle - Ginkgo Biloba. Book 1 (Vol 1-2). 400 p. Available at: http://www.scribd. com/doc/74555470/Nature-s-Miracle-Ginkgo-BilobaBook-1-Vol-1-2-B-M-Begovic-Bego

Benčat', F. (1982). Atlas rozšírenia cudzokrajných drevín na Slovensku a rajonizácia ich pestovania. [Atlas of the spread of foreign trees in Slovakia and the zoning of their cultivation]. $1^{\text {st }}$ ed. VEDA : Bratislava. $451 \mathrm{p}$. (maps), 359 p. [In Slovak]. ISBN 71-059-82

Benčat', T. (2009). Dendrológia a ekológia drevín. [Dendrology and ecology of woody plants]. TU : Zvolen. 225 p. [In Slovak]. ISBN 978-80-228-1996-1

Brenner, E.D., Katari, M.S., Stevenson, D.W., Rudd, S.A., Douglas, A.W., Moss, W.N., Twigg, R.W., Runko, S.J., Stellari, G.M., Mccombie, W.R., \& Coruzzi, G.M. (2005). EST analysis in Ginkgo biloba: an assessment of conserved developmental regulators and gymnosperm specific genes. BMC Genomics, 6. https://doi.org/10.1186/147-2164-6-143

Diamond, B.J., Shiflett, S.C., Feiwel, N., Matheis, R.J., Noskin, O., Richards, J.A., \& Schoenberger, N.E. (2000). Ginkgo biloba extract: Mechanisms and clinical indications. Archives of Physical Medicine and Rehabilitation, 81(5), 668-678. https://doi.org/10.1016/s0003-9993(00)90052-2

Fan, X.X., Shen, L., Zhang, X., Chen, X.Y., \& Fu, C.X. (2004). Assesing genetic diversity of Ginkgo biloba L. (Ginkgoaceae) populations from China by RAPD markers. Biochemical Genetics, 42, 269-278.

Flora of North America. (2020). [cit. 2021-11-05]. Available at: http://floranorthamerica.org/Ginkgo biloba

Han, S., Wu, Z., Jin, Y., Yang, W., \& Shi, H. (2015). RNA-Seq analysis for transcriptome assembly, gene identification, and SSR mining in ginkgo (Ginkgo biloba L.). Three Genetics \& Genomes, 11(37). https://doi.org/10.1007/s11295-015-0868-8

He, B., Gu, Y., Xu, M., Wang, J., Cao, F., \& Xu, L. (2015). Transcriprome analysis of Ginkgo biloba kernels. Frontiers in Plant Science, 6. https://doi.org/10.3389/fpls.2015.00819

Hrubík, P., Kollár, J., Rovná, K., Tkáčová, S., \& Mňahončáková, E. (2011). Kvalitatívna inventarizácia, klasifikácia a hodnotenie zdravotného stavu drevín pre účely záhradno-architektonickej a krajinárskej tvorby [Qualitative inventory, classification and evaluation of the health status of woody plants for the purposes of garden-architectural and landscape creation]. Nitra : Slovenská pol'nohospodárska univerzita v Nitre. [In Slovak]. ISBN 978-80-552-0651-6.

Jiang, L., You, R. L., Li, M.X, \& Shi, Ch. (2003). Identification of a Sex-Associated RAPD Marker in Ginkgo biloba. Journal of Integrative Plant Biology, 45(6), 742-747. 
Klečková, J. (2010). Jinan - relikt z období permu. Zahradnictví, 9. Available at: https://www.zahradaweb. cz/jinan-relikt-z-obdobi-permu/

Kleijnen, J., \& Knipschild, P. (1992). Ginkgo biloba. Lancet, 340, 1136-1139.

Kolař́k, J., Diensbír, F., Horáček, P., Praus, L., \& Reš, B. (2005). Péče o dřeviny rostoucí mimo les [Care of woody plants growing outside the forest]. $2^{\text {nd }}$ part. Vlašim: Český svaz ochránců přírody. [In Czech]. ISBN 8086327442.

Kwant, C. 2011. The ginkgo pages. Available at: http://kwanten.home.xs4all.nl/

Li, G.P., Zhang, C.Q., \& Cao, F.L. (2013). An efficient approach to identify Ginkgo biloba cultivars by using random amplified polymorphic DNA markers with a manual cultivar identification diagram strategy. Genetics and Molecular Research, 12(1), 175-182. https://doi.org/10.4238/2013.January.24.10

Li, Y.Y., Zang, L.P., \& Chen, X.Y. (2009). Development of polymorphic microsatellite markers for Ginkgo biloba L. by database mining. Conservation Genet Resour, 1, 81-83.

Liao, L., Liu, J., Yanxia, D., Qian, L., Xie, M., Qijiong, Ch., Huagun, Y., Qiu, G., \& Liu, X. (2009). Development and application of SCAR markers for sex identification in the dioecious species Ginkgo biloba L. Euphytica, 169, 49-55. https:// doi.org/10.1007/s10681-009-9913-8

Lin, Ch.P., Wu, Ch.S., Huang, Y.Y., \& Chaw, S.M. (2012). The complete chloroplast genome of Ginkgo biloba reveals the mechanism of inverted repeat contraction. Genome Biology and Evolution, 4(3), 374-381.

https://doi.org/10.1093/gbe/evs021

Lin, X., Zhang, J., Li, Y., Luo, H., Wu, Q., Sun, Ch., Song, J., Li, X., Wei, J., Lu, A., Qian, Z., Khan, I.A., \& Chen, S. (2011). Functional genomics of a living fossil tree, Ginkgo, based on next-generation sequencing technology. Physiologia Plantarum, 143, 207-218.

https://doi.org/10.1111/j.1399-3054.2011.01500.x

Mantovani, N.C., Grando, M.F., Xavier, A., \& Otoni, W.C. (2013). In vitro shoot induction and multiplication from nodal segments of adult Ginkgo biloba plants. Horticultura Brasileira, 31, 184-189.

Mei, Z., Khan, M.A., Zeng, W., \& Fu, J. (2014). DNA fingerprints of living fossil Ginkgo biloba by using ISSR and improved RAPD analysis. Biochemical Systematics and Ecology, 57, 332-337. https://doi.org/10.1016/j.bse.2014.09.007

Milewicz, M., \& Sawicki, J. (2013). Sex-linked markers in dioecious plants. Plant Omics Journal, 6(2), 144-149.

Mohanta, T.K. (2012). Advances in Ginkgo biloba research. genomics and metabolomics perspectives. African Journal of Biotechnology, 11(93), 15936-15944. https://doi.org/10.5897/AJB12.627

Pagan, J., \& Randuška, D. (1988). Atlas drevín [Atlas of trees]. $2^{\text {nd }}$ part. $1^{\text {st }}$ ed. Bratislava:Obzor. [In Slovak].

Raček, M., Lichtnerová, H., \& Dragúňová, M. (2009). Reakcie Ginkgo biloba L. na zmeny životných podmienok [Reactions of Ginkgo biloba L. to changes in living conditions]. In Zborník referátov z vedeckej konferencie s medzinárodnou účastou: "Dendrologické dni v Arboréte Mlyňany SAV 2009", Vieska nad Žitavou : Arborétum Mlyňany SAV. [In Slovak] ISBN 978-80-970254-4-1

Raček, M., Lichtnerová, H., \& Dragúňová, M. (2010). The influence of water scarcity on choosen physiological reactions of Ginkgo biloba L. seedlings. Acta horticulturae et regiotecturae, special Issue. Nitra : Slovak University of Agriculture in Nitra. p. 24-26.

Raček, M., Lichtnerová, H., \& Dragúňová, M. (2011). Reactions of Ginkgo biloba L. seedlings on water scarcity. Materials of the International conference 20-24 June 2011, Petrozavodsk, p. 257-260.

Ražná, K., \& Hrubík, P. (2016). Ginko dvojlaločné (Ginko biloba L.): genomická štúdia a kultúrne rozšírenie na Slovensku [Ginko biloba L.: genomic study and cultural distribution in Slovakia]. Nitra : SPU. [In Slovak] ISBN 978-80-552-1594-5

Ražná, K., Hrubík, P., Žiarovská, J., Kollár, J., Kullačová, D., Pavel, J., \& Štefúnová, V. (2014). Kultúrne rozšírenie ginka dvojlaločného (Ginkgo biloba L.) na Slovensku a hodnotenie jeho variability pomocou DNA markérov [Cultural distribution of Ginkgo biloba L. in Slovakia and evaluation of its variability using DNA markers]. Nitra : SPU. [In Slovak] ISBN 978-80-552-1231-9

Ražná, K., Sawinska, Z., Ivanišová, E., Vukovic, N., Terentjeva, M., Stričík, M., Kowalczewski, Pł., Hlavačková, L., Rovná, K., Žiarovská, J., \& Kačániová, M. (2020). Properties of Ginkgo biloba L.: Antioxidant characterization, antimicrobial activities, and genomic microRNA based marker fingerprints. International Journal of Molecular Sciences, 21(9), 3087. https://doi.org/10.3390/ijms21093087

Ražná, K., Žiarovská, J., Hrubík, P., Batyaneková, V., \& Vargaová, A. (2019). Ecologically conditioned imprinting of miRNA-based profiles of Ginkgo biloba L. growing in Slovakia. Folia Oecologica, 46(1), 54-62. https://doi.org/10.2478/foecol-2019-0008

Shen, L., Chen, X.Y., Zhang, X., Li, Y.Y., Fu, C.X., \& Qiu, Y.X. (2005). Genetic variation of Ginkgo biloba L. (Ginkgoaceae) based on cpDNA PCR-RFLPs: inference of glacial ferugia. Heredity, 94, 396-401. https://doi.org/10.1038/sj.hdy.6800616

Singh, B., Kaur, P., Singh, R.D., \& Ahuja, P.S. (2008). Biology and chemistry of Ginkgo biloba. Fitoterapia, 79, 401418. https://doi.org/10.1016/j.fitote.2008.05.007

Šmarda, P., Veselý, P., Šmerda, J., Bureš, P., Knápek, O., \& Chytrá, M. (2016). Polyploidy in a "living fossil" Ginkgo biloba. New Phytologist, 212, 11-14. https://doi.org/10.1111/nph.14062

Tekelová, D., Tóth, J., Mrlianová, M., Czigle, Sz., Filippová, D., \& Grančai, D. (2006). Stanovenie obsahu flavonoidov v jesenných listoch Ginkgo biloba L. kolorimetrickou a HPLC metódou [Determination of flavonoid content in autumn leaves of Ginkgo biloba L. by colorimetric and HPLC method]. Farmaceutický Obzor, 75(10-11), 272-279. [In Slovak] 
Tokár, F. (1968). Ginko dvojlaločné - pozoruhodný strom našich parkov [Ginkgo biloba - a remarkable tree of our parks]. Ochranca prírody a pamiatok, 8. [In Slovak].

Tokár, F. (1970). Pestovanie ginka na Slovensku [Ginkgo cultivation in Slovakia]. Záhradník, 4, 316-319. [In Slovak]

Tomaško, I. (2004). Historické parky a okrasné záhrady na Slovensku (História, lokalizácia, valorizácia, architektúra a spôsoby obnovy) [Historical parks and ornamental gardens in Slovakia (History, location, valorization, architecture and methods of restoration)]. Bratislava : VEDA. [In Slovak]. ISBN 80-224-0797-6.

Tommasi, F., \& Scaramuzzi, F. (2004). In vitro Propagation of Ginkgo biloba by using various bud cultures. Biologia Plantarum, 48, 297-300.

https://doi.org/10.1023/B:BIOP.0000033460.75432.d1

Van Beek, T.A. (2000). Ginkgo Biloba. Harwood Academic Publishers. p. 84. ISBN 90-5702-488-8. 532

Vreštiak, P., \& Osvald, Z. (1994). Všetko o ihličnanoch. [All about conifers]. Bratislava : Slovart. [In Slovak] ISBN 80-7445-126-6.

Wang, L., Zhao, J., Zhang, M., Weixin, L., Luo, K., Lu, Z., Zhang, Ch., \& Jin, B. (2015). Identification and characterization of microRNA expression in Ginkgo biloba L. leaves. Tree Genetics \& Genomes, 11(76), 1-18. https://doi.org/10.1007/s11295-015-0897-3

Wang, Y.Q., Shen, J.K., Berglund, T., Ohlsson, A.B., Tang, X.F., Zhou, Z.K., Wu, R.Y., Zhou, X.H., \& Chen, J.N. (2010). Analysis of expressed sequence tags from Ginkgo mature foliage in China. Tree Genetics \& Genomes, 6, 357-365. https://doi.org/10.1007/s11295-009-0254-5

Xie, Ch.X., Zhao, M.S., Fu, Ch.X., \& Zhao, Y.P. (2013). Development of the first chloroplast microsatellite loci in Ginkgo biloba (Ginkgoaceae). Applications in Plant Sciences, 1(8). https://doi.org/10.3732/apps.1300019
Xie, L., Hettiarachchy, N.S., Jane, M.E., \& Johnson, M.G. (2003). Antimicrobial activity of Ginkgo biloba leaf extract on Listeria monocytogenes. Journal of Food Science, 68, 268-270. https://doi.org/10.1111/j.1365-2621.2003.tb14150.x

Xu, M., Xu, L.A., Cao, F.L., Zhang, H.J., \& Yu, F.X. (2015). Development of novel chloroplast microsatellite markers for Ginkgo biloba. Genetics and Molecular Research, 14(3), 7715-7720. http://dx.doi.org/10.4238/2015.July.13.17

Yan, X.F., Lian, C.L., \& Hogetsu, T. (2006). Development of microsatellite markers in ginkgo (Ginkgo biloba L.). Molecular Ecology Notes, 6, 301-302. https://doi.org/10.1111/j.1471-8286.2006.01134.X

Yan, X.L., Chen, Y.Y., Guan, B.C., \& Fu, Ch.X. (2009). Eleven novel microsatellite markers developed from the living fossil Ginkgo biloba (Ginkgoaceae). Conserv Genet, 10, 12771279. https://doi.org/10.1007/s10592-008-9706-y

Zhang, Q., Li, J., Sang, Y., Xing, S., Wu, Q., \& Liu, X. (2015). Identification and characterization of microRNA in Ginkgo biloba var. epiphylla Mak. PLOS ONE, 10(5). https://doi.org/10.1371/journal.pone.0127184

Zhou, L., \& Wang, R. (2020). Analysis of Volatile Compounds from fresh and cooked Ginkgo biloba seeds. J. Science and Technology of Food Industry, 41(10), 238-243. https://doi.org/10.13386/j.issn1002-0306.2020.10.039

Zittlau, J. (2007). Liečivo ginkgo. Všetko o priaznivom účinku na telo, ducha a dušu [Ginkgo drug. All about the beneficial effect on the body, spirit and soul]. Bratislava: NOXI, s.r.o. [In Slovak] ISBN 978-80-89179-49-7. 